\title{
A RADIAL FOKKER-PLANCK MODEL FOR PLASMAS CONFINED BY MAGNETIC MIRROR FIELDS
}

A. H. Futch

May 1986

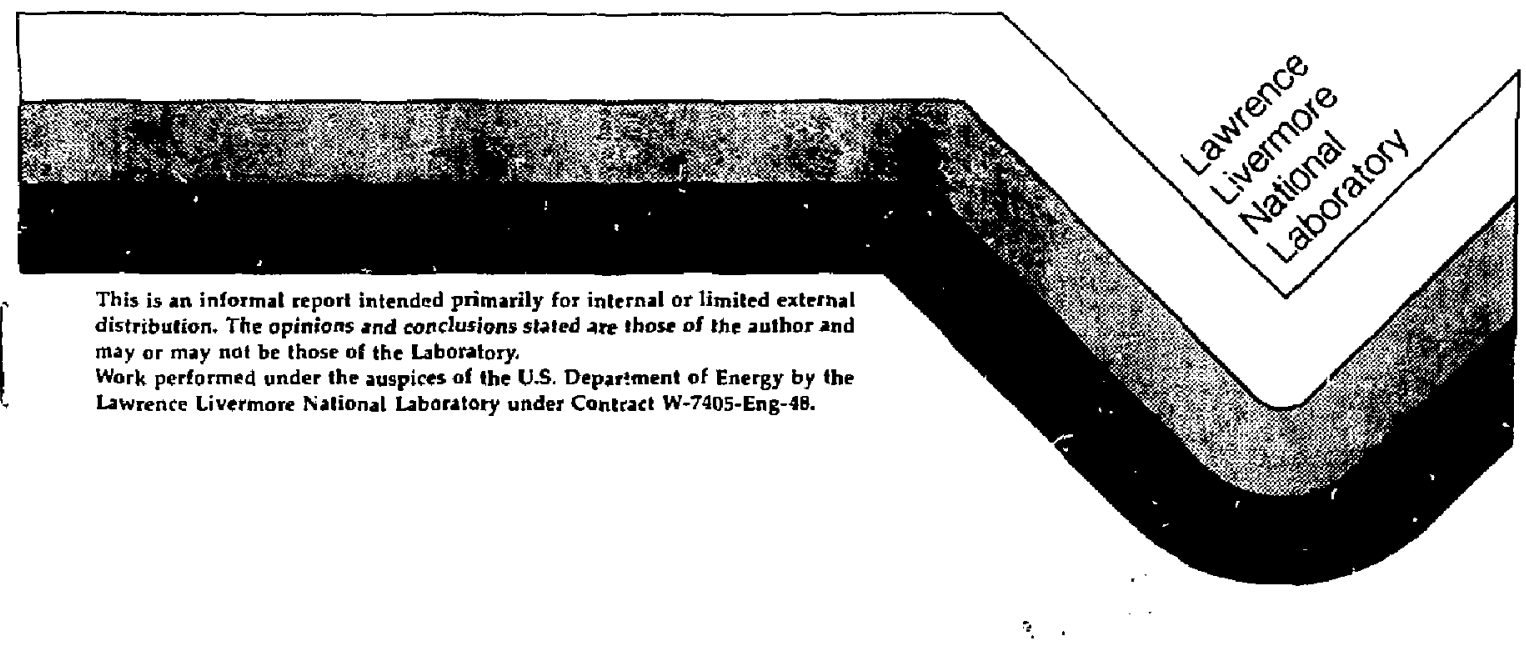

DIETRIBUTIOA UF THIS LCCLIHEAT IS UYLILIITE 


\section{A RADIAL FOKKER-PLANCK MDDEL FOR PLASMAS CONE INED BY MAGNETIC MIRROR FIELDS}

A. H. Futch

Lawrence $L i$ vermore National Laboratory Uni versity of Cal ifornia

Livermore, CA 94550

\section{DISCLAIMER}

This report wa propered as en accomat of wort sponsored by an afeacy of the United States Governmeat. Neither the Unitod State Government nor any apency thereof, nor any of their

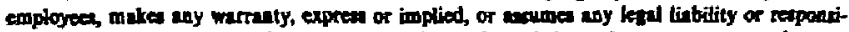

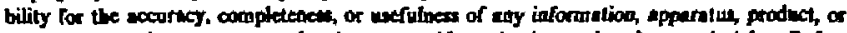

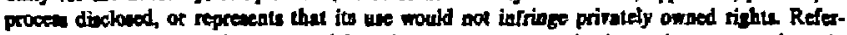

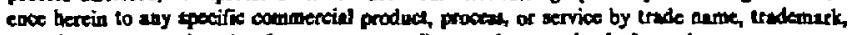

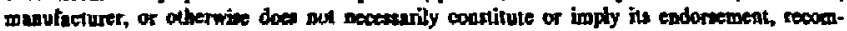
mendution, or favorint by the United State Gomeroment or any agency thereor. The vitw and opinions of anthors expresed berein do not necewarily wlate of reflect those of the United States Government or any afeacy thereof.

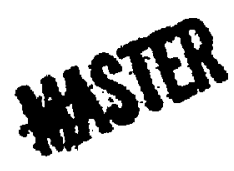


PREFACE

$\begin{array}{ll}\text { ABSTRACT } & 3\end{array}$

I. INTRODUCTION 5

II. Mathematical MODEL 7

1. Boltzmann's Equation 8

2. Atomic Physics Source and Loss Terms 20

3. The Electron Equations 26

4. Nelatral Gas Equations 28

5. Quasilinear Diffusion Terms 31

6. Complete Guiding Center Equation 36

III. NUMERICAL PROCEDURE 38

IV. COMPARISON WITH PREVIOUS WORK 43

$\begin{array}{ll}\text { REFERENCES } & 47\end{array}$

$\begin{array}{lll}\text { APPENDIX A } & \text { A1 }\end{array}$

APPENDIX B

$$
-i i i-i k
$$


A RADIAL FOKKER-PLANCK MODEL FOR PLASMAS CONFINED BY MAGNETIC MIRROR FIELDS

\author{
A. H. Futch \\ Lawrence Livermore National Laboratory \\ Undversity of Callforiala \\ Livermore, CA 94550
}

PREFACE

The work described in the present paper was inltiated almost ten years ago and was essentially completed in 1977 when quasilinear diffusion was added to tho existing two-dimensional Fokker-Planck eode for the distribution function, $f(v, r)$. An approximate treatment by T. B. Kalser had extended the one-dimensional quasilinear theory to include spatial diffusion in two dimensions perpendicular to the magnetic rield. Arter adding quasilinear terms to the radial Fokker-Planck code (RFP), and setting the riuctuating potential in the code equal to probe measurements of this potential in the fan region of the $2 \times 1$ IB experiment, calculated values of $T_{e}$ decreased from classical Fokker-Planck values (1000 eV for $2 X I I B)$ to the $50 \mathrm{eV}$ to $200 \mathrm{eV}$ range observed in $2 X I I B$ and TMX experiments. Other plasma parameters such as the particle confinement times, radial density profiles, ion densities and energies were also in good agreement. As a result of the r'elatively large gyro-radius, radial transport in the Fokker-Planck model was observed to dominate the pariciele and power loss from the plasma core. 
Prellminary results were discussed in an American Physical Society paper on Detober 30, 1978 at Colorado Springs, Colorado. The data irom this poster session is presented without conment as Appendix A in this paper. In addition the author (memorandum $A$. H. Futch, June 25, 1979) showed that if the ion lifetime in the 2XIIB experiment was limited by electron drag and if $T_{e}$ was constant or decreased with radius as measured experimentally, it followed from the drag fontrula that the ion Iffetim: decreases with radlus. However, calculations of the fon lifetime from measurements of the radial density profile and assuming the only sources was beam ionization resulted In an ion lifetime that increased with radius. This suggested to the author that electron drag was not the only important loss process and that radially outward diffusion due to beam trapping and fon cyclotron fluctuations could play an important role. This unexpected nt behavior vanished if an additional source term was introduced to account for radial transport. Appendix $C$ is another memorandum showing a comparison between the calculated radial plasma-density profile and experimentally measured results for the plug in the TMX experiment. The data illustrates the effects of radizl diffusion in creating a hollow density profile.

Since then the code has been used to calculate plasma parameters including radial transport in TMX, TMX-U, and in several other laboratories. This report is being issued in response to requests for description of the code and will serve to document the earlfer work. At the present time the code is being used for modeling bean injection in the central cell of the thermal barrier tandem mirror TMX-U. 
ABSTRACT

A time-dependent computer model has been developed for the spatially dependent distribution function $f(r, v, t)$. An orb1t averaged Fokker-Planck equation treats Coulomb collisions and varlous atomic physics processes. The motivation for the present code is to provide an accurate treatment of gyro-radius effects and realistlc beam geometries which have been neglected in previous Fokker-planch codes. Finite width beams may be offset from the plasma center to determine beam injection for bulld-up studies. The Inclusion of a finite gyro-radius is essential for studying radial transport due to both quas1linear fluctuations and charge exchange collisions. Charge exchange with energetic beam atoms and plasma erosion due to charge-exchange collisions with thermal gas are both included. These effects are major contributions to the loss of plasma in present experiments. Quasilinear diffusion terms include the effects of turbulent diffusion in the model. ${ }^{2}$ The model has been used to study particle and energy confinement times in the $2 X I I B$ experiment and in the mirror plug of the TMX experiment. Radial diffusion significantly changes the particle and energy confinement times in the plasma core. Calculated values of $T_{e}$ decrease from classical FokkerPlanck values (1000 eV for $2 X I I B)$ to the $50 \mathrm{eV}$ to $200 \mathrm{eV}$ range observed in 2XIIB and TMX experimerits. Radial density profiles and nt scaling with plasma radius ard magnetic field agree with experimental data. 


\section{INTRODUCTION}

The present code results from the merger of two separate lines of development: time-dependent Fokker-Planck codes describing Coulomb collision processes, and spatially dependent particie bulldup codes. Fowler and Hankin (1962) and Futch and Killeen (1962) solved the Fokker-Planck equations for both ions and electrons with the assumption that the FokkerPlanck equation is separable. The evolution of numerical Fokker-Planck calculations was reviewed by Kuo-Petravic et al. in 1969. The timedependent code developed at LLNL and descrlbed in detail by Killeen and Futch (1968) ineluded the effects of charge exchange and $t$ ime dependent buildup of plasmas formed by neutral-beam injection. The t1me-dependent LLNL code was extended to treat an arbitrary number of charged species (Eutch, Holdren, Killeen, and Mirin (1972)). Parallel development at LLNL resulted in the development of two-dimensional, $f(v, \theta)$, multi-species Fokker-Planck codes (J. Killeen and A. A. Mirin, 1978 and J. Killeen et al., 1976).

Spatially dependent particle buildup codes for plasma production by neutral-atom injection evolved from an early code at LLNL (Futch, Heckrotte, Damm, Killeell, and Mish, 1962). Mclr (unpublished) and Stallard (Stallard, UCRL-51784) studied the radial expansion (or erosion) caused by the competition between the trapping rate for ions at the plasia boundary and the 1 oss rate due to charge exchange with thermal gas at the boundary. Their code included the radial dependence of the thermal gas and the trapping of secondary beams of neutral atoms resulting from charge exchange collisions, but failed to distinguish between particle density and guiding 
center density in the trapping process. The present work generalizes the particle bui1dup code [Futch, Heckrotte, and Damm (1962)] to an -4. Aation for the distribution function $f(r, v, t)$, and includes to first order secondary neutral atom beams resulting frcm charge-exchange collisions cf plasma ions with thermal gas and beam neutrals. The Coulomb processes are described by extending the mult1-species Fokker-Planck code [Futch, Holdren, Killeen, and Mirin (t972)] to include the radial dimension.

Turbulent diffusion has been observed to play a key role ln many mirror experiments (Bajborodov, et al., "Control Fuslon and Plasma Physics," 2, 647 (1971); C. C. Damm, J. H. Foote, A. H. Futch, A. L. Gardner, and R. F. Post, Phys. Rev. Letters 13, 464 (1964); L. C. Kuo, E. G. Murphy, M. Peiravic, and D. R. Sweetman, Phys. Fluids, I, 988 (1964); F. H. Coensgen, W. F. Cummins, B. G. Logan, A. W. Molvik, W. E. Nexsen, T. C. Simonen, B. W. Stallerd, and W. C. Turner, "Stabilization of a Neutral-Beam-Sustained, Mirror-Confined Plasma," Phys. Rev. Lett. 35, 1501 (1975). Calculations by Berk and Stewart ${ }^{2}$ included quasilinear diffuston in velocity space to model DCLC turbulence in the 2XIIB experiment. Kaiser ${ }^{1}$ suggested an approximate treatment for retalning the turbulence model of the $1 \mathrm{D}$ theory and extending the velocity space dimensionality to two directions perpendicular to the magnetic field. Baldwin, Berk, and Pearlstein developed a quasilinear model for the nonlinear saturation of the DCLC mode in mirror machines which was consistent with experimental observations in 2xIIB. [D. E. Baldwin, H, L. Berk, and L. D. Pealstein, "Turbulent Lifetimes In Mirror Machines," Phys. Rev. [ett. 36, 1051 (1976).] We have included quasilinear diffusion In the present code using the formulation derived by Berk and Matsuda $(p, 103$, UCRL-50002-77). 
A principal contribution of the work is the inclusion of atonic physies trapping and loss processes including the finite orbit size of the lons in a two-d1mensional orb1t-averaged Fckker-Planck code, the code is capable of treating an arbitrary number of species and neutral beams of finite width. Diagnostics serve as a check on the conservation or particles and energy. They also display the deta1ls of particle and energy transfer within the plasma, and particle losses from the plasina. Finally, the good agreement with extensive experimental data, gives confidence in the use of the code for extrapolating to new regions.

The work is organized as follows; Section II describes the basic equations and assumptions. Section III discusses the computational procedure. A comparison with previous work is given in Section IV. The code results are compared with experimental data in Appendix $A$.

\section{MATHEMATICAL MODEL}

This section is organized as follows; the generalized Boltzmann equation is first expressed in terms of constants of the motion. Addition il simplirication is introduced by making the frequently used assumption of isotropy of the Rosenbluth potentials and by integrating the distribution over $\theta$ to obtain an equation independent of angle. The atomic physics and neutral gas squation are discussed in Sections 2 and 3 . The electron equation is introduced in Section 4 and the quasilinear terms in Section 5. The complete guiding center equation is discussed in the final section. 


\section{Boltzmann's Equation}

The Boltzmann equation with Fokker-Planck collision teras is the appropriate kinetic equation for describing the distribution function for charged particles in a magnetic field:

$$
\left.\left.\left.\frac{\partial r_{a}}{\partial t}+\underline{v} \cdot \frac{\partial f_{a}}{\partial \underline{r}}+\frac{E}{\pi_{a}} \cdot \frac{\partial f_{a}}{\partial \underline{y}}=\frac{\partial f_{a}}{\partial t}\right)_{c}+\frac{\partial r_{a}}{\partial t}\right)_{a}+\frac{\partial r_{a}}{\partial t}\right)_{q 1}
$$

where $f_{a}$ is the distribution function for particles of specie "a", $\left.\partial f_{a} / \partial t\right)_{a}$ repregents the atomic physics terms, and $\left.\partial f_{a} / \partial t\right)_{c}$ is the collision term. The Fokker-Planck collision term may be generalized to liclude turbulent diffusion by adding the appropriate quasilinear collision terms shown explicitly as the last term of Eq. (1). For solving Eq. (1) in mirror geometry, a spherical coordinate system is usually chosen for velocity space and a cylindrical coordinate system for physlcal spane with $z$ along the axis of symmetry and $\theta=0$ corresponding to the magnetic field direction. For the present model, the following assumptions are made:

1. The usual square weli model for the spat lal variation of the ambipolar potential and magnetic fleld is adopted where the distribution functions are independent of the spatial coordinate along the magnetic field (z direction). Fur this model which represents the plasma in the uniform cestral region of a long mirror machine the magnetic mirrors and ambipolar potential effect only the boundary conditions in velocity space. 
2. The only force acting on : particle other than collisions is the magnetic sorce resulting from a uniform magnetic fleld.

3. The distribution function is a function of radius, but independent of the azimuthal angle in physical space.

Under these conditions, Eq. (1) reduces to

$$
\left.\left.\left.\frac{\partial f_{a}}{\partial t}+v_{r} \frac{\partial f_{a}}{\partial r}=\frac{\partial f_{a}}{\partial t}\right)_{c}+\frac{\partial f_{a}}{\partial t}\right)_{a}+\frac{\partial f_{a}}{\partial t}\right)_{q l}
$$

Equation (2) may be simplified by observing that the second term on the left hand side is larger by the ratio $\Omega / v_{c}$ than the remaining terms where $\Omega$ is the ion cyclotron frequency and $v_{c}$ is the coulomb collision frequency. This suggests an expansion of $f_{a}$ in powers of $v_{c} / n$. Dropping the subscript "a", one sets

$$
r=r_{0}+r_{1}+\cdots
$$

and equates like powers of $v_{c}$ in to obtain

$$
\begin{aligned}
& v_{r} \frac{\partial f_{0}}{\partial r}=0 \\
& \left.\left.\left.\frac{\partial f_{0}}{\partial t}+v_{r} \frac{\partial f_{1}}{\partial r}=\frac{\partial f_{0}}{\partial t}\right)_{c}+\frac{\partial f_{0}}{\partial t}\right)_{a}+\frac{\partial f_{0}}{\partial t}\right)_{q l}
\end{aligned}
$$


From Eq. (4), the zeroth order distribution function is independent of $r$. In general, this means that

$$
f_{0}=f_{0}\left(E, \mu, P_{X}, t\right)
$$

Where $E$, the particle energy, $\mu$, the magnetlo moment, and $P_{X}$, the canonical angular momentum, are all constants of the motion. For the square well model, E may be replaced by $v, \mu$ by $\theta$, and $P_{X}$ by the guiding center radius, R. It follows from the formulae for $\mu$, and $P_{X}$ that $\theta$ and $R$ are constants of the motion if the magnetic field $B$ is constant, i.e.,

$$
\mu=\frac{E_{1}}{B}
$$

and

$$
P_{x}=\frac{e B}{2}\left(R^{2}-\rho^{2}\right)
$$

where $p$ is the Larmor radius. The zero order distribution function becomes

$$
f_{0}=r_{0}(v, \theta, R, t)
$$

Dividing Eq. (5) by $v_{r}$ and integrating around a gyro-orbit gives 


$$
\begin{aligned}
& \left.\left.\left.\frac{\partial f_{0}}{\partial t} \oint \frac{d r}{v_{r}}+\oint \frac{\partial f_{i}}{\partial r} d r=\oint\left[\frac{\partial f_{0}}{\partial t}\right)_{c}+\frac{\partial f_{0}}{\partial t}\right)_{a}+\frac{\partial f_{0}}{\partial t}\right)_{q l}\right] \frac{d r}{v_{r}} \\
& \left.\left.\left.\frac{\partial f_{0}}{\partial t}=\frac{1}{\tau} \oint\left[\frac{\partial f_{0}}{\partial t}\right)_{c}+\frac{\partial f_{0}}{\partial t}\right)_{a}+\frac{\partial f_{0}}{\partial t}\right)_{q l}\right] \frac{d r}{v_{r}}
\end{aligned}
$$

where

$$
\tau=\oint \frac{d r}{v_{r}}=\frac{2 \pi}{\Omega}
$$

The Fokker-Planck collision term (Rosenbluth et al., 1957) is

$$
\begin{aligned}
& \left.\frac{\partial f_{a}}{\partial t}\right)_{c}=\frac{1}{2} \nabla_{v} \cdot\left[\left(\nabla_{v} f_{a}\right) \cdot D-f_{a} \nabla_{v} h_{a}\right] \\
& =\frac{1}{2 v^{2}} \frac{\partial}{\partial v}\left\{v^{2}\left[D_{v v} \frac{\partial f_{a}}{\partial v}+\frac{D_{v \theta}}{v} \frac{\partial f_{a}}{\partial \theta}+\frac{D_{v \phi}}{v \sin \theta} \frac{\partial f_{a}}{\partial \phi}-f_{a} \frac{\partial h_{a}}{\partial v}\right]\right\} \\
& +\frac{1}{2 v \sin \theta} \frac{\partial}{\partial \theta}\left\{\sin \theta\left[D_{\theta v} \frac{\partial f_{a}}{\partial v}+\frac{D_{\theta \theta}}{v} \frac{\partial f_{a}}{\partial \theta}+\frac{D_{\theta \phi}}{v \sin \theta} \frac{\partial f_{a}}{\partial \phi}-\frac{f_{a}}{v} \frac{\partial h_{a}}{\partial \theta}\right]\right\} \\
& +\frac{1}{2 v \sin \theta} \frac{\partial}{\partial \phi}\left\{D_{\phi v} \frac{\partial f_{a}}{\partial v}+\frac{D_{\phi \theta}}{v} \frac{\partial f}{\partial \theta}+\frac{D_{\phi \phi}}{v \sin \theta} \frac{\partial f_{a}}{\partial \phi}-\frac{f_{a}}{v \sin \theta} \frac{\partial n_{a}}{\partial \phi}\right\}
\end{aligned}
$$

where 


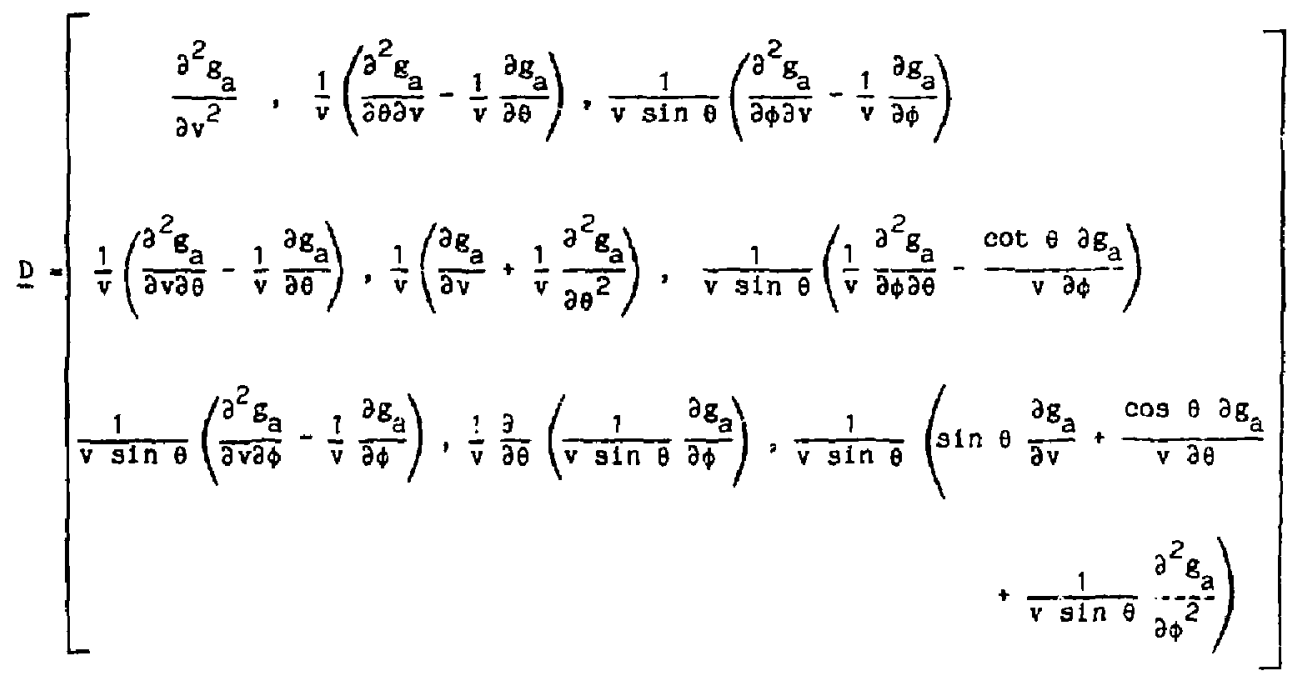

and the Rosenbluth potentials $\mathrm{B}_{\mathrm{a}}$ and $\mathrm{b}_{\mathrm{a}}$ are given $b_{\mathrm{j}}$

$$
\begin{aligned}
& B_{a}=\sum_{b} \frac{4 \pi z_{a}^{2} z^{2} e^{4 l} l n \Lambda}{m_{a b}^{2}} \int f_{b}\left(\underline{v}^{\prime}\right)\left|\underline{v}-\underline{v}^{\prime}\right| d \underline{v}^{\prime} \\
& h_{a}=\sum_{b}^{4 \pi z_{a}^{2} z_{b}^{2} e^{4} 1 n A_{a b}} \frac{\left(m_{a}+m_{b}\right)}{m_{a}^{2}} \int \frac{f_{b}\left(\underline{r}^{\prime}\right)}{\mid \underline{\underline{v}}-\underline{v}^{\prime} T} d \underline{\underline{v}}^{\prime}
\end{aligned}
$$

The formulation for the Coulorab logarithm used here has been discussed previously (Futch, Holdren, etc. 1972). We make the frequently used assumption of isotropy for $g_{a}$ and $h_{a}$ (Ben Danfel and Allis (1962) Fowler and Rankin (1962), Futch and KHlieen (1962), Kuo-Petravic et al. (1969)). With the 1sotropy assumption i.e., 


$$
\frac{\partial g_{a}}{\partial \theta}=\frac{\partial h_{a}}{\partial \theta}=\frac{\partial g_{a}}{\partial \phi}=\frac{\partial h_{a}}{\partial \phi}=0
$$

the tensor $\underline{D}$ greatly simplifies to
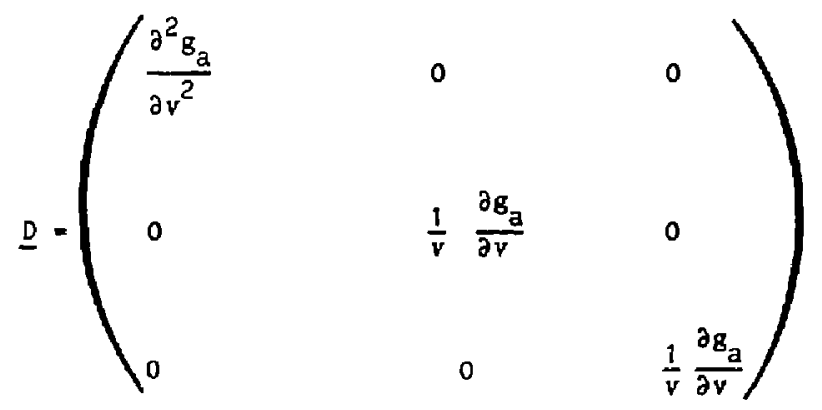

and $\mathrm{Eq}$. ( 14 ) becomes

$$
\begin{aligned}
\left.\frac{\partial f_{a}}{\partial t}\right)_{c}= & -\frac{1}{2 v^{2}} \frac{\partial}{\partial v}\left[2 v^{2} \frac{\partial h_{a}}{\partial v} f_{a}-\frac{\partial}{\partial v}\left(v^{2} f_{a} \frac{\partial^{2} g_{a}}{\partial v^{2}}\right)+2 \frac{\partial g_{a}}{\partial v} f_{a}\right] \\
& +\frac{1}{2 \sin \theta} \frac{\partial}{\partial \theta}\left[\frac{\sin \theta}{v^{3}} \frac{\partial g}{\partial v} \frac{\partial f_{a}}{\partial \theta}\right] \\
& \left.+\frac{1}{2 v \sin \theta} \frac{\partial}{\partial \phi}\left[\frac{1}{v^{2} \sin \theta} \frac{\partial g_{a}}{\partial v} \frac{\partial f_{a}}{\partial \phi}\right]+\frac{\partial f_{a}}{\partial t}\right)_{q l} .
\end{aligned}
$$

Changing the coordinate $\phi$ to the constant of motion coordinate $R$ gives 


$$
\begin{aligned}
\left.\frac{\partial \mathrm{f}}{\partial t}\right)_{c}= & -\frac{1}{v^{2}} \frac{\partial}{\partial v}\left[\left(v^{2} \frac{\partial h_{a}}{\partial v}+\frac{\partial g_{a}}{\partial v}\right) s_{a}-\frac{\partial}{\partial v}\left(\frac{v^{2} f_{a}}{2} \frac{\partial^{2} B_{a}}{\partial v^{2}}\right)\right] \\
& +\frac{1}{2 \sin \theta} \frac{\partial}{\partial \theta}\left[\frac{g \ln \theta}{v^{3}} \frac{\partial g_{a}}{\partial v} \frac{\partial f_{a}}{\partial \theta}\right] \\
& \left.+\frac{1}{2 R} \frac{\partial}{\partial R}\left[\frac{1}{v^{3} \sin ^{2} \theta} \frac{r^{2} v_{r}^{2}}{\Omega^{2} R} \frac{\partial g}{\partial v} \frac{\partial f}{\partial R}\right]+\frac{\partial f}{\partial t}\right)_{q l}
\end{aligned}
$$

where we have used the relation

$$
R^{2}=r^{2}+\rho^{2}-2 r \rho \cos (\pi / 2+\phi)
$$

with $\phi$ the angle between $v_{1}$ and the $r$ vector, $\hat{e}_{r}$, to obta in

$$
\frac{\partial}{\partial \phi}=\frac{\partial R}{\partial \phi} \frac{\partial}{\partial R}=\frac{r v}{\Omega R} \frac{\partial}{\partial R} .
$$

Substituting the collision term into Eq. (11) and integrating around a gyro-orbit gives

$$
\begin{aligned}
& \frac{\partial f_{a}}{\partial t}=-\frac{1}{v^{2}} \frac{\partial}{\partial v}\left[f_{a}\left(v^{2}\left\langle\frac{\partial h}{\partial v}\right\rangle+\left\langle\frac{\partial g}{\partial v}\right\rangle\right)-\frac{\partial}{\partial v}\left(\frac{v^{2} f_{a}}{2}\left\langle\frac{\partial^{2} B_{a}}{\partial v^{2}}\right\rangle\right)\right] \\
& +\frac{1}{2 \sin \theta} \frac{\partial}{\partial \theta}\left[\frac{\sin \theta}{v^{3}}\left\langle\frac{\partial g}{\partial v}\right\rangle \frac{\partial f}{\partial \theta}\right] \\
& +\frac{1}{2 R} \frac{\partial}{\partial R}\left[\frac{1}{v^{3} \Omega^{2} R \sin ^{2} \theta}\left\langle r^{2} v_{r}^{2} \frac{\partial g}{\partial v}\right\rangle \frac{\partial f_{a}}{\partial R}\right]+\left\langle\frac{\partial f}{\partial t}\right\rangle_{q l}+\left\langle\frac{\partial f_{a}}{\partial t}\right\rangle_{a p}
\end{aligned}
$$


In Eq. (23), $f_{a}$ is the zero order distribution function for species "an and $\langle$ represents an average around the orbit, l.e.,

$$
\left\langle>=\frac{1}{\tau} \oint() \frac{d r}{v_{r}}\right.
$$

where $t$ is the orbit time. Multiplying Eq. (23) by $K_{a} \sin \theta d \theta$ and integrating over $\theta$ from $\theta_{L C}$ to $\pi-\theta_{L G}$, one obtains an -quation independent of $\theta$, i.e.,

$$
\begin{aligned}
\frac{\partial F}{\partial t}= & -\frac{1}{v^{2}} \frac{\partial}{\partial v}\left[\frac{1}{v^{3} \Omega^{2} \theta \sin ^{2} \theta}\left\langle r^{2} v_{r}^{2} \frac{\partial g}{\partial v}\right\rangle \frac{\partial F}{\partial R}\right] \\
& \left.+\left[\frac{K_{a} \sin \theta_{L C}}{v^{3}}\left\langle\frac{\partial g_{a}}{\partial v}\right\rangle \frac{\partial F}{\partial \theta}\right)_{\theta L C}\right]+\frac{1}{R} \frac{\partial}{\partial R}\left[\frac{1}{2 v \Omega^{2} R}\left\langle r^{2} \cos ^{2} \phi \frac{\partial g}{\partial v}\right) \frac{\partial F a}{\partial R}\right] \\
& +\left(\frac{\partial F a}{\partial t}\right)_{q l}+\left\langle\frac{\partial F a}{\partial t}\right\rangle_{a p}
\end{aligned}
$$

where

$$
F_{a}(v, R, t)=K_{a} \int_{\theta_{L C}}^{\pi-\theta_{L C}} f_{a}(v, \theta, R, t) \sin \theta d \theta
$$

The normalization constant, $K_{a}$, is determined by the initial density and distribution function for each species, i.e.,

$$
n_{a}(R, 0)=K_{a} \int_{0}^{\infty} F_{a}(v, R, 0) v^{2} d v
$$


In deriving Eq. (25) from Eq. (23) we have replaced tne $\theta$ depencient gyroradius $p(\theta)$ by

$$
\langle\sin \theta\rangle=\frac{\int \sin ^{2} \theta E(\theta) d \theta}{\int F(\theta) \sin \theta d \theta}
$$

to obtain the orbit-averaged coefflclents in $\mathrm{Eq}$. (25).

To evaluate the second term on the right hand side of Eq. (25) requires a knowledge of $\partial f_{a}(\partial \theta)_{\theta_{L C}}$. In general, the $\theta$ dependence of $f$ is an unknown quantity which may be represented by an infinite series of normal mode solutions which are functions of the mirror ratio $R_{m}$. In aractice, the ambipolar potential $\$$ has been taker into account by substiluting for $R_{m}$ an effective mirror ratio, Reff, defined by

$$
R_{\text {eff }}=R_{m}\left(l \pm \frac{\Phi}{E}\right)^{-1}
$$

which enters as $\log _{10} R_{\text {eff }}$ in the expression for the plasma lifetime. The normal mode distribution may be varied from a broad distribution in a to a "highly peaked" distribution by changing $R_{m}$. In the present model, the error resulting from the uncertainty in the angular dependence of the distribution function has been investigated by running the code for angular distributions varying from a broad normal mode distribution to a "highly peaked" distribution. The error (whlch will be discussed in a later section) resulting from the uncertainty in the $\theta$ dependence of the distribution function 18 small. This fortunate circumstance results from the fact that normal mode distributions for mirror ratios in the range from 
1 to 5 are already well peaked and going to a more highly peaked or $\delta$-function does not change the particle lifetime significantly. The procedure followed is to pick an angular distribution and perform the orbit average Integrals in Eq. (25) using the chosen angular distribution and an average $p$ calculated using Eq. (28). In practice we pick either the usual normal mode distribution or a highly peaked angular distribution if quasilinear processes are experted to dominate the diffusion processes. Substituting for the Rosenbluth potentials and noting that the guiding center density, $D(R, v, t)$ is related to the lowest order distribution function $F(R, v, t)$ by $2 \pi F_{a}(R, v, t)=D_{a}(R, v, t), E q .(25)$ may be expressed in terns of the guiding center density as

$$
\left.\left.\frac{\partial D_{a}}{\partial t}(R, v)=\frac{1}{v^{2}} \frac{\partial}{\partial v}\left[A_{a} D_{a}+B_{a} \frac{\partial D}{\partial v}\right]-\frac{C a}{v^{2}} D_{a}+\frac{1}{R} \frac{\partial}{\partial R}\left[E \frac{\partial D_{a}}{\partial R}\right]+\frac{\partial D_{a}}{\partial t}\right)_{q l}+\frac{\partial D_{a}}{\partial t}\right)_{a p}
$$

The coefficients are defined as foliows:

$$
\begin{aligned}
& A_{a}=\frac{\Omega \Gamma_{a}}{2 \pi} \sum_{b}^{\sum} \oint\left[\left(\frac{Z_{b}}{Z_{a}}\right)^{2}\left(\frac{M_{a}}{M_{b}}\right) K_{b} \ln \Lambda_{a b} N\left(E_{b}\right)\right] \frac{d r}{v_{r}} \\
& B_{a}=\frac{\Omega \Gamma_{a}}{2 \pi} \sum_{b}^{\sum} \oint\left(\frac{Z_{b}}{Z_{a}}\right)^{2} K_{b} \ln \Lambda_{a b}\left[\frac{1}{3 v} E\left(E_{b}\right)+\frac{v^{2}}{3} M\left(E_{b}\right)\right] \frac{d r}{v_{r}}
\end{aligned}
$$




$$
\begin{aligned}
& c_{a}=c_{s}=\frac{a \Gamma_{a}}{2 \pi}\left(\lambda_{a} / 2 v\right) \underset{b}{\sum} \oint\left(\frac{Z_{b}}{Z_{a}}\right)^{2} K_{b} \ln \Lambda_{a b}\left[N\left(F_{b}\right)-\frac{1}{3 v^{2}} E\left(F_{b}\right)\right. \\
& \left.+\frac{2 v}{3} M\left(F_{b}\right)\right] \frac{d r}{v_{r}} \\
& c_{s} \leq c_{f} \\
& c_{a}-c_{p}=v^{2} v_{t} / L_{p}{ }^{R} \\
& c_{g}>C_{f} \\
& E_{a}=\frac{r_{a}}{4 \pi v a R} \sum_{b} \oint\left(\frac{Z_{b}}{z_{a}}\right)^{2} k_{b} \ln A_{a b}\left[r^{2}-\frac{\left(R^{2}-r^{2}-p^{2}\right)^{2}}{4 p^{2}}\right] \\
& x\left[N\left(E_{b}\right)-\frac{1}{3 v^{2}} E\left(E_{b}\right)+\frac{2 v}{3} M\left(F_{b}\right)\right] \frac{d r}{v_{r}}
\end{aligned}
$$

where $k_{v}$ is a normalization constant determined by the initial density and distribution for each species, i.e.,

$$
n_{b}(0)=k_{b} \int_{0}^{\infty} F_{b}(v, 0) v^{2} d v
$$

and

$$
\begin{aligned}
& L_{p}=\text { plasma length } \\
& v_{t}=\sqrt{\frac{Z_{a}{ }^{\phi}}{m_{a}}} \\
& M(F)=\int_{v}^{\infty} F(y, t) y d y
\end{aligned}
$$




$$
\begin{aligned}
& N(F)=\int_{0}^{v} F(y, t) y^{2} d y \\
& E(F)=\int_{0}^{v} F(y, t) y^{4} d y \\
& \Gamma_{a}=\frac{4 \pi z_{a}^{4} e^{4}}{m_{a}^{2}} \\
& \Omega=e B / m_{a} c=\frac{2 \pi}{\oint \frac{d r}{v_{r}}}
\end{aligned}
$$

i

and where we have used the emptrical fit of noberts and Capp (1960) for the coefficient of the scattering loss term in Eq. (25), 1.e.,

$$
\lambda_{a}=\frac{2 \sin \phi_{L C}}{f_{a}} \frac{\partial f_{a}}{\partial \theta}=-\left(\log _{10} R_{a}\right)^{-1}
$$

The "effective" mirror ratio, $R_{a}$, is defined by

$$
R_{a}=\frac{R_{m}}{1+Z_{a} e_{\phi} / E_{a}(v)}
$$

with $F_{m}$ equal to the vacuum mirror ratio, and $E_{a}(v)$ the ion energy. The definition used for the Coulomb logarithm, In $\Lambda_{a b}$ is discussed ir Ref. 6 .

The usual assumption in calculating the collisional loss rate for mirror confinement is that an ion scattered into the loss cone leaves the plasma immediately. This assumption is not valld for low energy lons where 
the transit time is longer than the collisional scattering time. Particle loss rates are expected to make a smooth transition from the usual mirror loss rate to trans1t time loss rates (Rognlien and Cutler, UCRL-84112). The coefflcient, $c_{a}$, of $\mathrm{Eq}$. (30) has been modifled to provide a smooth trangltion from mirror confinement to transit time confinement by replacing $c_{a}$ with the reclprocal of the transit time whenever $\tau_{r}>c_{a}^{-1}$.

\section{Atomle Physles Source and Loss Terms}

A major featurs of the present work is the retention of finite Larmor radius terms in the description of atomic physics processes. If an energetle beam of neutral atoms is injected through a plasma, the neutral beam atoms will become trapped in the plasma by ionizing collisions with both plasma ions and electrons, and by charge exchange collisions with plasma ions. If the injection is perpendicular to the magnetic axis, the trapped tons will move in a plane perpendicular to the confining field; and the trapping processes may be represented by a two-dimensional model. Figure 1 shows the geometry of the problem. The trapped ions move in ctrcular orbits of radius $\rho$. A neutral beam of width $w$ and displaced a distance $d$ above the magnetic axis passes through the confining field. The irapping and loss terms are descrlbed by

$$
\frac{\partial D_{a}(R, v)}{\partial t}=K_{2}^{a}(R, v)-K_{1}^{a}(R, v) D_{a}(R, v)-\Sigma_{k}\left(\sigma_{c x}^{k} v\right)\left\langle n_{0}(r)\right\rangle D_{a}(R, v)
$$

where 


$$
\left\langle n_{0}^{k}(r)\right\rangle=\frac{1}{\tau} \oint \frac{n_{0}^{k}(r) d r}{v_{r}}
$$

and $\sigma_{c x}^{k}$ is equal to elther the molecular charge exchange cross section, $\sigma_{c x}^{m}$ or the atomic charge exchange cross section $a_{c x}^{a}$ depending on the gas specles. The total trapping rate (londzation and charge-exchange) for species "a" is

$$
\begin{aligned}
k_{2}^{a}(R, v) & =\sum_{\alpha} \frac{\delta\left(v-x_{a}^{a}\right)}{2 \pi} \sum_{b} \frac{(a v)}{v_{a}^{\alpha}}\left[\int_{x_{2}}^{x_{3}} J_{a}^{a}(R, x) n_{b}(R, x) d x\right. \\
& \left.+\int_{x_{4}}^{x_{1}} J_{a}^{a}(R, x) n_{b}(R, x) d x\right] \frac{1}{4 \pi\left(v_{a}^{a}\right)^{2}}
\end{aligned}
$$

where $\mathrm{J}_{\mathrm{a}}^{\alpha}$ is the current per $\mathrm{cm}^{2}$ for beam comfonent a of spectes $\mathrm{a}$, and the factor $1 / 4 \pi\left(v_{a}^{\alpha}\right)^{2}$ occurs because the source is a delta function in velocity space. The integration over the angle $x$ in Eq. (33) accounts for the rractions of the azlthumal drift time within the beam, see F1g. 2 . The summations indlcated in Eq. (33) are over all beams ( g $_{\text {) }}$ and over all plasma species ( $(\bar{b})$. The a component of the beam velocity for specie "a" is $v_{a}^{\alpha}$. The second term of Eq. (31) corresponds to the loss of plasma ions by charge-exchange collisions with neutral beam atoms. $K_{i}^{a}(R, v)$ is defined by

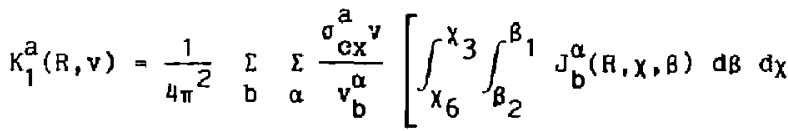

$$
\begin{aligned}
& \left.+\int_{X_{4}}^{x_{5}} \int_{B_{2}}^{\beta} J_{b}^{\alpha}(R, x, B) d B d x\right]
\end{aligned}
$$


The integration over the angle $\beta$ accounts for the fraction of the Byromotion within the beam and the integration over $x$ accounts for the fraction of azimuthal drift time within the beams. See. Fig. 4.

The last term of Eq. (31) is the loss of particles by charge-exchange collisions with thermal gas. The Integration variables in Eqs. (33) and (34) are detalled in the following way.

$$
\begin{array}{lll}
h_{1}-w / 2+d & u_{1}=\left(h_{1}-\rho\right) / R & u_{3}=\left(h_{1}+\rho\right) / R \\
h_{2}=w / 2-d & u_{2}=\left(h_{2}+\rho\right) / R & u_{5}=\left(h_{2}-\rho\right) / R
\end{array}
$$

then

$$
\begin{array}{rlrl}
x_{1} & =\sin ^{-1} u_{1} & -1<u_{1}<1 \\
& =-\pi / 2 & u_{1} \leq-1 \\
& =\pi / 2 & u_{1} \geq 1 \\
x_{2}=\pi-x_{1} & \\
x_{3}=\pi+\sin ^{-1} u_{2} & u_{2}<1 \\
x_{3}=3 / 2 \pi & u_{2}>1 \\
x_{4}=3 \pi-x_{3} & u_{3}>1
\end{array}
$$




$$
\begin{array}{ll}
x_{5}=\sin ^{-1} u_{3} & u_{3}<1 \\
x_{5}=\pi / 2 & u_{3}>1 \\
x_{6}=\pi-x_{5} & \\
x_{7}=\pi+\sin ^{-1} u_{r} &
\end{array}
$$

Let

$$
\begin{aligned}
& u_{4}=\frac{|R \sin x|-n}{\rho} \quad \text { where } n=n_{1} \quad 0 \leq x \leq \pi \\
& =h_{2} \quad \pi<x \leq 2 \pi \\
& \alpha=\cos ^{-1} u_{4} \\
& \text { If }\left|u_{4}\right|<1 \text {, then } \\
& B_{1}=\alpha+\pi / 2-x \quad 0 \leq x \leq \pi \\
& B_{1}=\alpha+3 / 2 \pi-x \quad \pi<x \leq 2 \pi \\
& B_{2}=B_{1}-2 \alpha \\
& \text { If }\left|u_{4}\right|>1 \\
& B_{1}=2 \pi
\end{aligned}
$$


$B_{2}=0$

$\alpha=\pi$

The attenuation of the primary neutral beams in passing through the plasma 18 given by

$$
J_{a}^{\alpha}(r, x)-J_{a}^{\alpha}(x, y) \exp -\left(\sum_{b} \frac{(v v) \tau}{v_{a}^{\alpha}} \int_{x_{1}}^{x} n_{b}(x, y) d x\right)
$$

with

$$
x_{1}=-\sqrt{R_{p}^{2}-y^{2}}
$$

and $k_{p}$ the plasma radius.

In the foregoing equations, (ov) $)_{T}$ indlcates the sum of the ov's for ionization by ions and electrons and for resonant charge-exchange collisions. The relative velocity between the two colliding particles is $v$. The cross sections are represented by analytical fits to experimental data. Expressions given by Riviere (Nuclear Fusion 11, 363 (1971)) are used for the resonant charge exchange and proton lonfzation cross sections. The ionization cross sections have been generalized by appropriate mass and $\mathbf{z}$ dependence to model ionizing collisions by other lon species. The cross sections $\sigma_{e x}^{a}$ and $\sigma_{i}^{a}$ are the atomic charge-exchange and ionization cross section for hydrogen atoms, respectively. They are def $t$ ned as follows: 
$\sigma_{c x}^{a}=\frac{0.6937 \times 10^{-14}\left[1-0.155 \log _{10}\left(\frac{E}{P}\right)\right]^{2}}{1+0.1112 \times 10^{-34}\left(\frac{E}{P}\right)^{3.3}} \mathrm{~cm}^{2} \quad(E$ in $\mathrm{eV}$

$$
\begin{aligned}
a_{1}^{a}=z_{10}^{2}-0.8712\left[\log _{10}(E / P)\right]^{2}-8.156 \log _{10}(E / P) & +34.833 \\
E & \leq 150 \times 10^{3} \mathrm{eV}
\end{aligned}
$$

$\sigma_{i}^{a}=\frac{3.6 \times 10^{-12} \log _{10}[0.1666(\mathrm{E} / \mathrm{P})] \mathrm{z}^{2}}{\mathrm{E} / \mathrm{P}}$

E > $150 \times 10^{3} \mathrm{eV}$

(37b)

where

$$
\begin{aligned}
& P=\text { the atomic mass } \\
& z=\text { the atomic number. }
\end{aligned}
$$

The foregoing cross sections do not apply to electrons. For electron fonization, we assume that the electron velocity is much greater than the ion velocity and that the distribution function is Maxwellian. Under these conditions the electron ionization rate paraneter may be represented by the following equations which are a function of the average electron energy only

$$
\overline{(0 v)_{1 e}}=A\left[1-\exp \left(-0.00074 E^{2}\right)\right]+B E-C E^{2} \quad E \leq 450 \mathrm{eV}
$$


where

$$
\begin{aligned}
& A=2.9297 \times 10^{-8} \\
& B=0.246862 \times 10^{-10} \\
& C=5 \times 10^{-14}
\end{aligned}
$$

$$
\overline{(\sigma v)_{1 e}}=\frac{A}{E^{1 / 2}}(\ln E+B)
$$

where

$$
\begin{aligned}
& A=1.62 \times 10^{-7} \\
& B=-2.0618
\end{aligned}
$$

\section{The Electron Equations}

Since the electrons differ only slightly from a Maxwellian distribution (Futch, Killeen, Holdren, Mirin, 1972) and have negligible Larmor radius, Pastukhov rate equations (Pastukhov, Nuclear Fusion 14, 3, 1974) with source terms are solved for the electron density, $n_{e}(R)$, and temperature, $T_{e}(R)$. The equation for $n_{e}(R)$ is 


$$
\begin{aligned}
\frac{d n_{e}(R)}{d t}= & \sum \sum_{b} \sum_{b} J_{b}^{a}(R, X) \lambda_{1}(R) \sum_{B} \frac{(\sigma V)_{i}}{v_{b}} n_{B}(R) \\
& +\sum_{B} \sum_{k}^{\sum} n_{B}(R) n_{0}^{k}(R)(O V)_{1}-\frac{n_{e}^{2}(R)}{n \tau}
\end{aligned}
$$

with

$\lambda_{1}=\frac{1}{\pi}\left[\sin ^{-1} n_{1} / R+\sin ^{-1} n_{2} / R\right]$

$(\sigma v)_{1}=$ the appropriate or for tonization by the piasma spectes

$$
\begin{aligned}
(n T)^{-1} & =\frac{2}{\sqrt{\pi}} \frac{n_{e}^{2}(R)}{n(R)_{T}} \frac{2 R_{m}}{2 R_{m}+1} \frac{\exp \left(-\frac{e \phi}{T}\right)}{\ln \left(4 R_{m}+2\right)} \frac{T_{e}}{e \phi}+\frac{T_{e}}{2 e \phi}-\frac{1}{4} \frac{T_{e}}{e \phi}+\ldots \\
T_{S} & =\frac{\sqrt{m} T^{3 / 2}}{\sqrt{2} \pi n e^{4} \lambda_{c}}
\end{aligned}
$$

where $\lambda_{c}$ is the Coulomb logarlthm. The first term on the right hand side or Eq. (40) is the source of electrons due to neutral beam trapping and the second term is the electron source which results fron tonization of thermal gas species $n_{0}^{k}(R)$. 
The electron energy equation is

$$
\begin{gathered}
\frac{d}{d t}\left[n_{e} E_{e}\right]=4 \pi \int_{0}^{\infty} \sum v_{e b} F_{e}(v) v^{2} d v+J_{e} E_{i n j} \\
-\frac{\left(\phi+I_{e}+E_{I}\right) n_{e}^{2}}{n \tau}
\end{gathered}
$$

where $\mathrm{E}_{1 \mathrm{nj}}$ 1s the energy at which electrons are injected Into the plasma. The firgt term of Eq. (42) is the energy transferred to the electrons frum the ion specles. Here

$v_{e b}=\frac{4 \pi z_{b}^{2} e^{4} l n \Lambda_{e b}}{m_{e}} K_{e} K_{b}\left[\int_{v}^{\infty} F_{b}(y, t) y d y-\frac{m_{e}}{m_{b}} \int_{0}^{v} F_{b}(y, t) y^{2} d y\right]$

where $K_{e}$ and $K_{b}$ are the normalizing constants determined by lihe initial density and distribution for each specles. The energy injected by the beam electrons is $\mathrm{J}_{e} \mathrm{E}_{i n j}$. The two loss terms are the power lost by electrons scattered into the loss-cone and the power lost by ionization of cold gas. $E_{I}$ is the energy required to ionize the neutral species and $\left(\phi+T_{e}\right)$ is the energy carried out by the hot electron which leaves when a cold electron is produced. The formula for $\mathrm{n} \tau$ is given by Eq. (41).

\section{Neutral Gas Equations}

Moiecular gas impinging on the plasma surface is converted into hydrogen Ions and a flux of neutral atams with energies of several el (Franck-Condon atoms) within a thin boundary layer at the plasita surface. 
Our model which neglects both the molecular gas and thermal ions resulting from the molecular dissociation assumes a radially directed flux of FranckCondon atons is created at the plasma surface. As these atoms penetrate the plasma, they undergo ionizing and charge-exchange collisions with plasma particles. The charge-exchange collision result in the formation of an additional flux of neutral atons at the plasma for energy which is in turn attenuated as it penetrates the plasma.

The radially dependent denstites of Franck-Condon and charge exchange neutral atoms is given by

$$
\mathrm{n}_{0}^{\mathrm{FC}}(\mathrm{R})=\mathrm{n}_{0}^{\mathrm{FC}}\left(\mathrm{R}_{\mathrm{p}}\right) \exp \left[-\sqrt{2} \int_{\mathrm{R}}^{\mathrm{R} p} \sum_{\mathrm{b}} \mathrm{n}_{\mathrm{b}} \frac{(\sigma \mathrm{V})_{\mathrm{T}}}{\mathrm{v}_{\mathrm{FC}}} \mathrm{dR}\right]
$$

and

$n_{0}^{C X}(R)=n_{0}^{F C}\left(R_{p}\right)(R A T I O) \exp \left[-\sqrt{2} \int_{R}^{R} \sum_{b}^{P} n_{b} \frac{(\sigma v)_{T}}{v_{c x}} d R\left(1-\exp -\sqrt{2} \int_{R}^{R} \sum_{R} n_{b} \frac{(\sigma v)_{T}}{v_{F C}} d R\right)\right]$

respectively, where

$$
\text { RATIO }=0.5\left[1+\left(R_{p}-R\right) / R_{p}\right]\left(v_{F C} / v_{c x}\right) \frac{\int_{R}^{R} \sum_{b} n_{b}(o v)_{c x} d R}{\int_{R}^{R} \sum_{b} n_{b}(o v)_{T}^{F C} d R}
$$

and, 


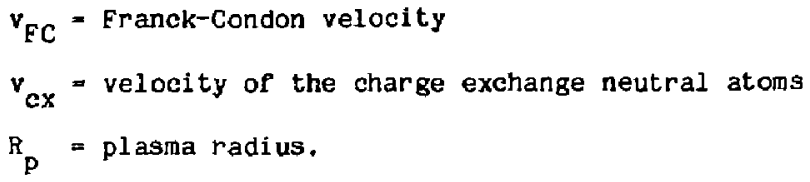

So far we have assumed that the neutral gas densities are independent of the axial coordinate $z$ as would be the case for a plasma modeled by a unlform cylinder. We now generalize these results to model a non-uniform plasma column with a varying elliptical cross section in the axial drection. We model the $z$-dependent plasma by a gaussian profile, 1.e.,

$$
\left.n^{a}(R, 2)=n^{a}(R) e^{-(z / L}\right)^{2}
$$

when $L_{p}$ is the plasma e-foiding lets:th. We define $f(z)$ as the minor axis scale length to the midplane scale length. We can then replace $d R$ in equations (45) through (47) with $f(z)$ dR to obtain the increased gas penetration along the thin side of the fan region. We then write the following expression for the radially dependent and z-averaged gas densities

$$
\left\langle n_{0}^{k}(R)\right\rangle=\frac{\int_{0}^{z_{m}} e^{-\left(z / L_{p}\right)^{2}} n_{0}^{k}(R) \frac{d z}{(B(z)}}{\int_{0}^{z m} e^{-\left(z / L_{p}\right)^{2}} \frac{d z}{B(z)}}
$$

For secondary beam components resulting from charge-exchange with plasma lons, the model deseribed by Stallard (UCRL-51784) has been used. 
The generalized form of his equations becomes

$$
\begin{aligned}
\frac{d J_{a}(-z)}{d z}= & n_{a} \sum_{b} \frac{\left.\sigma_{a} v\right) c x}{v_{a}}\left[F_{b, a} J_{b}(-z)+\left(1-F_{b, a}\right) J_{b}(z)\right] \quad(a \geq 2) \\
& +n_{a} \sum_{b}\left(\sigma_{a} v\right)_{c x} n_{b}(-z) \\
& -\frac{J_{a}(-z)}{v_{a}}\left[n_{a}(\sigma v)_{1}+n_{a}(0 v)_{c x}\left(1-F_{a, a}\right)+\sum_{b=a} n_{b}(a v)_{T}\right] \\
& +\left[1-F_{a, a}\right] n_{a}(0 v)_{c x} \frac{J_{a}(z)}{v_{a}}
\end{aligned}
$$

The $\mathrm{F}_{\mathrm{a}, \mathrm{b}}$ 's are the forward scattering fraction deseribed by Stallard. Equation (50) is solved for the range $-R_{W} \leq z \leq R_{W}$. The secondary beams are included in the summations for the trapping and loss terms discussed earlier; see Eqs. (33) and (34).

\section{Quasilinear Diffusion Terms}

Berk (UCRL-80272) has derived the quasilinear diffusion equation of a plasma in a magnetic field. His theory takes into account spatial inhomogeneity of the magnetic field and includes the orbit and bounce motion of particles in a magnetic mirror field. A form of Berk's equation for the guiding center density in cylindrical ccordinates is used here (Berk and 
Matsuda - unpublished). The quastlinear diffusion terms were derived by using an expansion in the Larmor radius for the limit where the bounce frequency is less than the variation of the cyclotron frequency sampled by a particle. The quasilinear diffuston terms for the guiding center density become

$\left.\frac{\partial D_{a}^{\prime}(R, \theta, v)}{\partial t}\right)_{q l}=\left(2 m \omega_{c l} \frac{\partial}{\partial v^{2}}+\frac{\ell}{\omega_{c 1}} \frac{1}{B} \frac{\partial}{\partial R}\right) D_{v}\left(v^{2}, R\right)\left(2 m \omega_{c 1} \frac{\partial}{\partial v^{2}}+\frac{\ell}{\omega_{c 1}} \frac{\partial}{\partial R}\right) D_{a}^{\prime}(R, \theta, v)$

In Eq. (51), $D_{a}^{\prime}(R, y, \theta)$ is the guiding center distribution function; 1 and $m$ are azimuthal and harmonic mode numbers.

The quasilinear diffusion coefficlent, $D_{v}$, is

$$
D_{v}=\sum_{m}\left(\frac{e \bar{\phi}_{m}(R)}{m_{a}}\right)^{2} J_{m}^{2}\left(k_{\perp} v_{\perp} / w_{c i}\right) \Omega_{m}^{-1}
$$

where $\bar{\phi}_{\mathrm{m}}(r)$ is the perturbed potential and $n_{\mathrm{m}}^{-1}$ is the correlation time given by

$$
\Omega_{m}^{-1}=\frac{\Delta \omega}{\left(\omega-m_{c 1}\right)^{2}+\Delta t}
$$

To obtain terms independent of $\theta$, we muliply by $\sin \theta d \theta$ and integrate from $\theta_{L C}$ to $\pi-\theta_{L C}$ as before. The result_ng equation for the quasilinear terms may be put in the form 
$\left.\frac{\partial D_{a}^{(R, v)}}{\partial t}\right)_{q 1}=\frac{1}{v^{2}} \frac{\partial}{\partial v}\left[A_{q 1} D_{a}+B_{q 1} \frac{\partial D}{\partial v}\right]-\frac{C q 1}{v^{2}} D_{a}+\frac{1}{R} \frac{\partial}{\partial R}\left[E_{q 1} \frac{\partial D}{\partial R}+F_{q I} D_{a}+G_{q 1}\right]$

In Eq. (54),

$$
D_{a}(R, v)=\int_{\theta_{L C}}^{\pi-\theta} L C D_{a}^{\prime}(R, \theta, v) \sin \theta d \theta
$$

and the quasllinear coefficients are defined as follows:

$$
\begin{aligned}
& A_{q l}=v \int D_{v}(v \sin \theta) \sin ^{2} \theta \cos \theta \frac{1}{D_{a}} \frac{\partial D_{a}^{\prime}}{\partial \theta} d \theta+v \frac{m l}{R} \frac{\partial D_{v}}{\partial R} \sin \theta D_{a}^{\prime} / D_{a} d \theta \\
& B_{q l}=v^{2} \int D_{v}(v \sin \theta) \sin ^{3} \theta D_{a}^{\prime} / D_{a} d \theta \\
& C_{q l}=\left.2 D_{v}\left(v \sin \theta_{L C}\right) \sin \theta_{L C} \cos ^{2} \theta_{L C} \frac{1}{D_{a}} \frac{\partial D_{a}^{\prime}}{\partial \theta}\right|_{\theta_{L C}}+\frac{m \ell}{R}\left(\alpha^{\prime}=B^{\prime}+v Y^{\prime}\right) \\
& E_{Q I}=\frac{\ell^{2}}{\omega_{C i^{2}}^{R}} \alpha \\
& E_{q l}=\frac{m l}{v}\left[\frac{2 \beta}{v}+\gamma+\frac{\delta}{v}\right] \\
& C_{q l}=\frac{2 m !}{v} \frac{\partial D a}{\partial v} \alpha
\end{aligned}
$$


with

$$
\begin{aligned}
& \alpha=\int D_{v} \sin \theta D_{a}^{\prime} / D_{a} d \theta \\
& B=\int D_{v} \cos \theta \frac{\partial D_{a}}{\partial R} \frac{\partial^{2} D_{a}^{\prime}}{\partial \theta \partial R} d \theta \\
& \gamma=\int \frac{\partial D_{v}}{\partial v} \sin \theta D_{a}^{\prime} / D_{a} d \theta \\
& \delta=\int \frac{\partial D_{v}}{\partial \theta} \cos \theta D_{a}^{\prime} / D_{a} d \theta \\
& a^{\prime}=\int \frac{\partial D_{v}}{\partial v} \sin \theta D_{a}^{\prime} / D_{a} d \theta \\
& \beta^{\prime}=\int \frac{\partial D_{v}}{\partial R} \cos \theta \frac{1}{D_{a}} \frac{\partial D_{a}^{\prime}}{\partial \theta} d \theta \\
& \gamma^{\prime}=\int \frac{\partial^{2} D_{v}}{\partial R \partial v} \sin \theta D_{a}^{\prime} / D_{a} d \theta
\end{aligned}
$$

If the real part of $\omega$ is near a harmonic of $\omega_{c i}$, then $n_{m}=\Delta \omega$. The Bessel functions of Eq. (52) are rapldiy varylng as the particle moves along its trajectory. To avoid the numerical difficulties associated with using Eq. (52) In a numerical code, an analytio expression representing the 
envelope of the oscillationg has been used by previous workers (Berk and Stewart, UCRL-T8407) and is adopted here. The square of the Bessel function is represented by

$$
J_{m}^{2}(z)=\frac{F_{m}(z) H(z)}{F_{m}(z)+H(z)}
$$

with

$$
F_{m}(z) *\left[(z / 2)^{m} / m !\right]^{2}
$$

and

$$
H(z)=(\pi z)^{-1}
$$

The radial dependence of $\phi_{m}^{-2}(R)$ is modeled by the following function which vanishes at the origin and therefore has a zero particle flux at $R=0$ :

$$
F(R)=\frac{(1+\alpha) R^{2}}{R^{2}+a R_{p}^{2}}
$$

with $\alpha$ an input number, one obtains.

$$
\frac{\partial D_{v}}{\partial R}=\frac{2 \alpha R_{p}^{2}}{(1+\alpha) R^{3}} \mathscr{F}(R) D_{v}
$$


$-36-$

Using Eq. $(60), \alpha^{\prime}, \beta^{\prime}$, and $\gamma^{\prime}$ may be rewritten as

$$
\alpha^{\prime}=\frac{2 \alpha R_{p}^{2}}{(1+\alpha) h^{3}} \mathscr{T}(\mathrm{R}) \alpha
$$

$$
\begin{aligned}
& \beta^{\prime}=\frac{2 \alpha \mathrm{R}_{\mathrm{P}}^{2}}{(1+\alpha) \mathrm{R}^{3}} \mathscr{T}(R) B \\
& \gamma^{\prime}=\frac{2 \alpha \mathrm{R}_{\mathrm{P}}^{2}}{(1+\alpha) \mathrm{R}^{3}} \mathscr{F}(\mathrm{R}) Y
\end{aligned}
$$

6. Complete Guiding Center Equation

Substituting in the quasilinear and atomic physics terms, Eq. (30) may be rewritten as

$$
\begin{aligned}
\frac{\partial D_{a}(R, v)}{\partial t}= & \frac{1}{v^{2}} \frac{\partial G_{a}}{\partial v}-\frac{C a}{v^{2}} D_{a}+\frac{1}{R} \frac{\partial}{\partial R}\left[H_{a}+G_{q 1}\right]+K_{2}^{a}(R, v) \\
& -K_{1}^{a}(R, v) D_{a}-\sum_{K}(\sigma v)_{o x}^{k}\left\langle n_{o}^{k}(r)\right\rangle D_{a}
\end{aligned}
$$

(be)

where 


$$
\begin{aligned}
& G_{a}=A_{a} D_{a}+H_{a} \frac{\partial D_{a}}{\partial v} \\
& H_{a}=F_{q l} D_{a}+E_{a} \frac{\partial D_{a}}{\partial R}
\end{aligned}
$$

and the coefficients $A_{a}, B_{a}, C_{a}$. and $E_{a}$ have been redefined to include the quasilinear coefficlents, l.e.,

$$
\begin{aligned}
& A_{a}=A_{a}+A_{q l} \\
& A_{a}=B_{a}+B_{q l} \\
& C_{a}=C_{a}+C_{q l}
\end{aligned}
$$

and

$$
E_{a}=E_{a}+E_{q l}
$$

Equation (62) contains velocity diffusion terms and radial diffusion terms as well as space and velocity dependent source and loss terns. For a spatially uniform plasma, the guidfng cencer equation reduces to the FokkerPlanck of Futch et al. (1972) with the quasilinear terms set equal to zero and when the transit time correction to the mirror 1099 rate is negligible, i.e., coefficient $\mathrm{C}$ of Eq. (62) becomes the mirror loss rate ondy. 
III. NUMERICAL PROCEDURE

Equation (62) is solved by a two step process. The time-dependent change due to radial diffusion is solved first, i.e.,

$$
\left.\frac{\partial D_{a}}{\partial t}(R, v)\right)_{r}=\frac{1}{R} \frac{\partial}{\partial R}\left[H_{a}+G_{q 1}\right]
$$

Integrating Eq. (65) over the volume gives

$$
\left.\int_{R=0}^{R=R} p \frac{\partial D_{a}}{\partial t}(R, v)\right)_{r} R d R=0
$$

as long as $H_{a}=G_{q l}=0$ for $R=0$ and $R=R_{p}$. At the origin, this condition reduces to $\partial D_{a} / \partial R=0$ since the form of $\phi_{m}^{-2}$ requires $F_{q l}=G_{q l}=0$ for $R=0$ and $\partial D_{a} / \partial R=0$ at the origin. For $R=R_{p}$ the appropriate boundary condition is $H_{a}+G_{Q I}=0$ for the radial flux to vanish. The boundary condition applied in the present model is $D_{a}(R, V)=0$ for $R+\langle\sin \theta\rangle v / \Omega=0$ where $\langle\sin \theta\rangle$ is averaged over the angular distribution. The radial grid is sufficiently large sucin that the wall loss 1 s negligible. The radial flux to the wall is monitored by a term in the conservation equations discussed in the next section.

Equation (65) may be written as an implicit difference equation and then expressed as the linear algebraic system 


$$
-\alpha_{L}^{n+1} D_{L+1}^{n+1}+\beta_{L}^{n+1} D_{L}^{n+1}-\gamma_{L}^{n+1} D_{L-1}^{n+1}=\delta_{L}^{n}
$$

as discussed in Ref. 6. The indictes $n$ and $L$ are the time and spatial Indicies, respectively. Equation (67) is solved using the standard tridiagonal method of Richtmyer and Morton (1967), generalized to vector equations.

The solution of Eq. (65) is expressed in derivative form and added to Eq. (62) as a spatially dependent source term tn place of the radial derlvative terms. The resulting equation may be written in dimensionless form as

$\left.\left.\frac{\partial D_{a}}{\partial t}=\frac{t}{x^{2}} \frac{\partial G_{a}}{\partial x}-\frac{C}{x^{2}} D_{a}+\frac{\partial D_{a}}{\partial t}\right)_{r}+K_{2}^{a}-K_{1}^{a} D_{a}-\sum_{k} \sigma_{c x}^{m} v_{0} x<n_{0}^{k}(r)\right\rangle D_{a}$

with $x=v / v_{0}$. In the absence of source and loss terms the particle density, $n_{a}$, should remaln constant in time. This condition gives the appropriate boundary condition for the solution of Eq. (68). That is

$$
\frac{d n_{a}}{d t}=k_{a} \int_{0}^{x \max } \frac{j}{x^{2}} \frac{\partial}{\partial x}\left[A_{a} D_{a}+B_{a} \frac{\partial D_{a}}{\partial x}\right] x^{2} d x=0
$$

or

$$
\left[A_{a} D_{a}+B_{a} \frac{\partial D_{a}}{\partial X}\right]_{X=0}^{X=X_{\max }}=0
$$


for $t>0$. For $X=0$, the boundary condition reduces to $2 D_{a} / \partial X=0$ since $A_{a}$ is zero at the origin.

Equation (68) is solved by the same procedure outlined above for Eq. (65) and discussed in Appendix $B$ of Ref. 6.

At each time step, Eq. (67) is solved simultaneously with the electron equations. The particle diatribution function, $F_{a}$, is obtained from the transformation

$$
F_{a}(x, r, t)=\frac{2}{\pi} \int_{|R-\rho|}^{R+\rho} \frac{D_{a}(x, R, t) R d R}{\sqrt{4 R^{2} r^{2}-\left(R^{2}+r^{2}-\rho^{2}\right)^{2}}}
$$

The number density and average energy of each ion species are calculated from the equations

$$
\begin{aligned}
& n_{a}(r, t)=k_{a} \int_{0}^{\infty} F_{a}(x, r, t) x^{2} d x \\
& \left.\left.E_{a}(r, t)=\frac{1}{2} m_{a} v_{0}^{2} \int_{0}^{\infty} F_{a}\right) x, r, t\right) x^{4} d x / \int_{0}^{\infty} f_{a}(x, r, t) x^{2} d x
\end{aligned}
$$

for each radial location. The plasma potential is computed for each lime step of the calculation by requirisg charge neutrality. A critical velocity $x_{\mathrm{cr}}(t)$ is determined such that electrons with $x<x_{\mathrm{cr}}(t)$ are not. lost and those w1th $x>x_{c r}(t)$ can be lost by scattering into the loss cone. At each 
time step and radial location the electron density is compared to the tatal ion charge density and the velocity $x_{\text {cr }}$ modifled accordingly.

We define $\mathrm{Q}^{-}$as the electron charge density and $\mathrm{Q}^{+}$as the total ion charge density. The potentlal $\phi$ is determined as followg. For each time step and radial location $Q^{-}(t)$ and $Q^{+}(t)$ are computed, the old electron density $Q^{-}(\tau-\Delta \tau)$ is compared to the old ion density, $Q^{+}(\tau-\Delta \tau)$, and the change In the electron density is compared to that of the lons. Since charge neutrality is to be maintained, if $Q^{-}(\tau-\Delta \tau)\{\$\}_{Q}^{+}(\tau-\Delta \tau)$ and $\Delta Q^{-}\left\{\leqslant \mid \Delta Q^{+}\right.$, the ambipolar potential is \{Increased/decreased by a small amount, and the time step is repeated. This cycling is continued until the condition $Q^{-}\left(\frac{3}{\underline{L}}\right)_{\Delta Q^{+}}$is obtained. The time dependent cross sections and the neutral gas equations are then solved before advancing to a new time step.

\section{Conservation of Particles and Energy}

A valuable feature of the present numerical model is the separate evaluaton of particle and energy conservation equations. Conservation equations provide a check on the numerical accuracy of the code, and they are Indispensable in providing a physlcal understanding of the complex interactions occurring in a spatially dependent Fokker-Planck code.

The particle equation for congervation of guiding center denslties at $R$ for species "a" is obtalned by integrating Eq. (62) over $v^{2} d y$ to obtain 


$$
\begin{aligned}
& \left.K_{a} \int \frac{\partial D_{a}}{\partial t}(R, v) v^{2} d v-\sum_{a} \int K_{2}^{1}(R, v) v^{2} d v+K a \int C_{a} D_{a} d v+K K_{a} \int \frac{\partial D_{a}}{\partial t}\right)_{r} v^{2} d v \\
& +K_{a} \int K_{1}^{a}(R, v) D_{a} v^{2} d v-\sum_{a} \int K_{2}^{c x}(R, v) v^{2} d v+\frac{2}{\pi} K_{a} \sum_{l}(\sigma v)_{c x}^{1}\left\langle n_{l}\right\rangle \int D_{a} v^{2} d v \\
& + \text { boundary } 1099=0
\end{aligned}
$$

where $K_{2}^{1}$ and $k_{2}^{c x}$ are the Ionlzation and charge exchange components of $k_{2}^{\text {a }}$ respectively. The velocity integrals are over the range from $v=0$ to $v_{\text {max }}$. The neutral gas dens1ty, $n_{\ell}$, for species " $\ell$ " averaged around the particle orbit is calculated by

$$
\left\langle n_{\ell}\right\rangle=\frac{2}{\pi} \oint \frac{n_{0}^{\ell}(r) r d r}{\left[4 R^{2} r^{2}-\left(R^{2}+r^{2}-\rho^{2}\right)^{2}\right]^{1 / 2}}
$$

here integration limits are from $|R-p|$ to $R+p$. The last term of $E_{q} .(73)$ represents particles that diffuse spatially beyond the plasma radius and are lost from the calculation. The number of particles lost in this manner are monitored by adding up the number of particles at each radial position after solving the gulding center equation but before the radius boundary conditions are applied and subtracting the particle sum after the radial boundary conditions are applied.

The energy conservation equation at radius $R$ is obtained by multiplying Eq. (62) by $k_{a} m_{a} v^{4} d v / 2$ and integrating to obtain 


$$
\begin{aligned}
& \sum_{a} \int_{a} \frac{m_{a} K a}{2} \int \frac{\partial D_{a}(R, v)}{\partial t} v^{4} d v-\sum_{a} \int K_{2}^{a}(R, v) E_{a}^{\alpha} v^{2} d v+\frac{m_{a} K_{a}}{2} \int C_{a} D_{a} v^{2} d v \\
& \left.+\frac{m_{a} K_{a}}{2} \int \frac{\partial D a}{\partial t}\right)_{r} v^{4} d v+\frac{m_{a} K_{a}}{2} \quad K_{1}^{a} D_{a} v^{4} d v-\frac{m_{a}}{2} \sum_{a} \int K_{2}^{c x}(R, v) v^{4} d v \\
& \left.+\frac{m_{a}{ }^{K} a}{\pi} \sum_{l}(\sigma v)_{c x}^{l}\left\langle n_{\ell}\right\rangle \int D_{a} v^{4} d v+\text { Energy lost to wall }\right\}+\frac{m_{a}{ }^{K} a}{2} \quad c_{e} F_{e} d v \\
& +n_{e} \sum_{l} \sigma_{1 e}^{l} r n_{2}\left(E_{1}^{2}+T_{e}\right)=0
\end{aligned}
$$

where $E_{I}^{\ell}$ is the energy lost in lonization of neutral specleg "l". The energy conservation equation has been summed over all ion specles; the last two terms of Eq. (75) are the energy loss terms for electrons. Since Eq. (75) Includes all species, energy transfer between specles sum to zero. Equationg (73) and (75) are Integrated over the plasma yolume to give the global particle and energy equations.

IV. COMPARISON WITH PREVIOUS WORK

To provide additional checks on code validity, results were compared with previous calculations. The atomic physics source and ioss terms were compared with Stallard's bulld-up code (B. W. Stallamd, UCRL-51784 (1975)). For this comparison, the calculated values of $n \tau$, Ion and electron energies, etc. from the present code were used as inputs for the bulld-up code. The results of this comparison at the plasma center (1.e., B-0) are as follows: 
RFP

particles/co-sec
Build-up

particles/co-sec
Difference

5

Total trapplng (ionization

plus charge exchange)

$2.63 \times 10^{14}$

$? .47 \times 10^{14}$

6.3

Net trapping (lonization) $1.13 \times 10^{14}$

$1.14 \times 10^{14}$

0.9

In general, a comparison of RFP code results with previous FokkerPlanck calculations requires the "turning-off" of additional physies not Included in previous results. Starting with a typical Fokker-Planck run for 2XIIB, quasilinear diffusion, thermal gas, half and third energy beam components were set equal to zero, and the gyro-radius was decreased by increasing the magnetic field. Figure 4 shows that the electron temperature on axis $(r-\phi)$ approached the classical Fokker-Planck of approximately 0.09 $E_{i}$ as additional physics terms ware turned of $f$ and the gyro-radius, $a_{i}$ ' decreased. The primary reason $T_{e}$ decreases is because the plasma confinement time $\tau_{p}$ decreases and since $T_{e}$ is proportional to $T_{p}^{2 / 5}, T_{e}$ decreases. Figure 5 compares the effective $n \tau, n \tau$ eff, as calculated fron the RFP code with results from two prevlous Livermore Fokker-Planck codes, the Multi-Species Code ${ }^{6}$, and the Hybrid-1 Code ${ }^{9}$. $n \tau_{\text {eff }}=n^{2} / \mathrm{J}$ where $n$ is the plasma density and $f$ is the trapping rate (particles/cm ${ }^{3}-s e c$ ); both measured on axis $(r=0)$. Because collisions are averaged around a particle orbit, a particle samples lower densities (for plasmas with a negative gradient) and $\mathrm{nt}_{\text {eff }}$ is larger for lower magnetfc fields. Therefore, the RFP results are plotted versus $1 / B$ for comparison with the zero gyro-radius results of previous codes. For this comparison, the additional physics 
terms not included in earlier Fokker-Planck codes are also set equal to zero as discussed earlier. The comparisons are within the 10 percent grid error experted from the various codes. The agreement 1 mproved when the number of velocity mesh points was doubled from 40 to 80 .

\section{Acknowl edgments}

The authors would like to acknowledge the early suggestion by Cinarles Damm that resonance charge exchange with injected beam atoms could lead to radíal transport. We would like to thank Ira Bernstein for driving the Coulorb cross-field diffusion term and for many hours of helpful discussion. Art Mirin provided the basic Fokker-planck package in a form sultable for the present code. The authors appreciate the many helpful discussions with the LLNL theory group including Don Pearlstein, Tom Rognlien, Herb Berk and others. Finally we would like to thank Bill Turner for his review of the manus $c r i p t$. 
REFERENCES

1. T. B. Kalser, MFE/TC/77-104 (unpublished).

2. H, L. Berk and J. J. Stewart, UCRL -78407 (1976).

3. T. K. Fowler and M. Rankin, Plasma Phys. (J. Nucl. Energy Part C) ㄴ, $311(1962)$.

1. A. H. Futch and J. K1lleen, UCRL-10294 (1962).

5. L. G. Kuo-Petravic, M. Petravic, and C. J. H. Watson, Proc. Int. Conf. on Nucl. Fusion Reactors, Culham, England, Paper 2.4 (1969).

6.' J. Killeen and A. H. Futch, J. Comput. Phys. 2, 236 (1968).

7. A. H, Futch, J. P. Holdren, J, Killeen, and A. A. Mirin, Plasma Phys. (J. Nuci. Energy Part C) 14, 211 (1972).

8. J. Killeen and K. D. Marx, Methods in Computational Physics. (Eds. Alder, Fernbach, and Rotenberg), 9, 422 (1970).

9. J, Killeen, et al., Methods in Computational Physics, (Academic Press, New York) 16, 389 (1976).

10. J. Killeen and A. A. Mirin, Theoretical and Computational Plasma Physies, (IAEA, Vienna) 27 (1978).

11. A. H. Futch, et al., Phys. Fluids 5, 1277 (1962).

12. Ralph Moir (unpublished).

13. B, W. Stallard, UCRL -51784 (1975).

14. Bajborodoy, et a1., Control Fuslon and Plasma Physics 2, 647 (1971).

15. C. C. Damm, J. H. Foote, A. H. Futch, A. L. Gardner, and R. F. Post, Phys. Rev, Lett. 13, 464 (1964).

16. L. C. Kuo, E. G. Murphy, M. Petravic, and D. R. Sweetman, Phys. Fluids 7. $938(1964)$. 
17. F. H. Coensgen, et al., Phys. Rev. Lett. 35, 1501 (1975).

18. D. E. Baldwin, H. L. Berk, and L. D. Pearlstein, Phys. Rev. Lett. 36 , 1051 (1976).

19. H. L. Berk and Y. Matsuda, pg. 103, UCRL-50002-77.

20. M. N. Rosenbluth, w. M. MacDonald, and D. L. Judd, Phys. Rev. 107, 1 (1957).

21. D. J. BenDanlel and W. P. All1s, Plasma Phys. (J. Nucl. Energy Part C) 4. $31(1962)$.

22. Ira Bernstein (private communication).

23. J. E. Goberts and M. L. Carr, UCRL-5651-T (1960).

24. T. Rognlien and T. Cutler, UCRL-84112 (1980).

25. A. C. Hiviere, Nucl. Fusion 11, 363 (1971).

26. V. P. Pastukhov, Nucl. Fusion 14, 3 (1974).

27. H. L. Berk, UCRL-80272.

28. R. D. Richtmyer and K. W. Morton, Difference Methods for In1tial-Value Problems, Interscience, New York (1967). 
FIGURE CAPTIONS

Figure 1. Geometry of neutral-atom injection experiment.

Figure 2. Integration limits for source and loss terms.

Figure 3. Integration limlts corresponding to fraction of lon orbit within the beam.

Fizure 4. Comparison of the electron temperature on axis as additional physics terms are modified in the RFP code.

Figure 5. Comparison of $n t$ with previous results. 
A. H. Futch - Figure 1

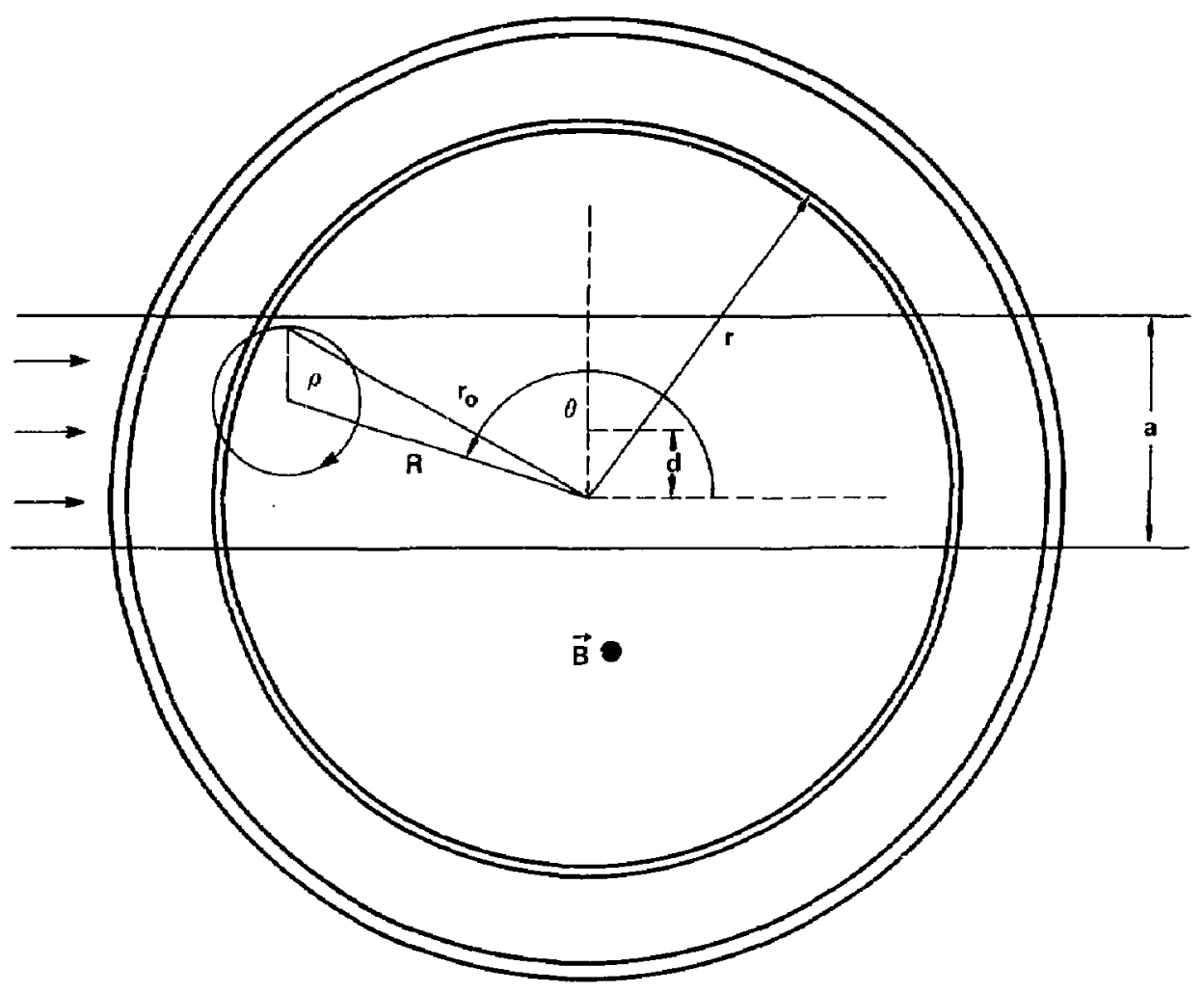


A. H. Futch - Eigure?

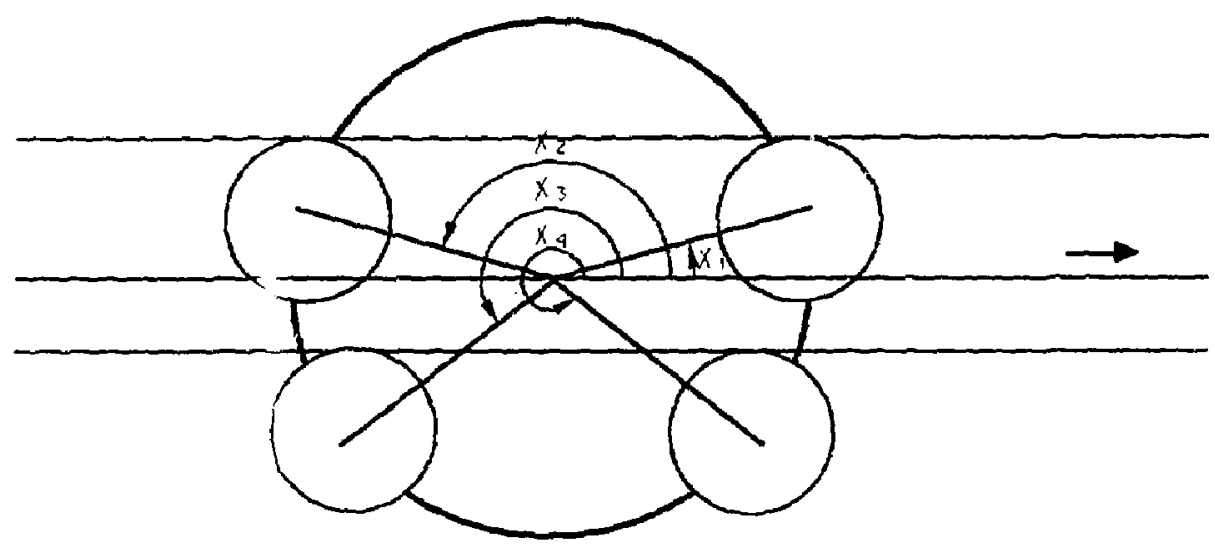

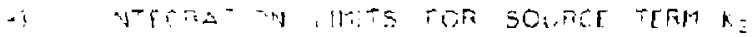

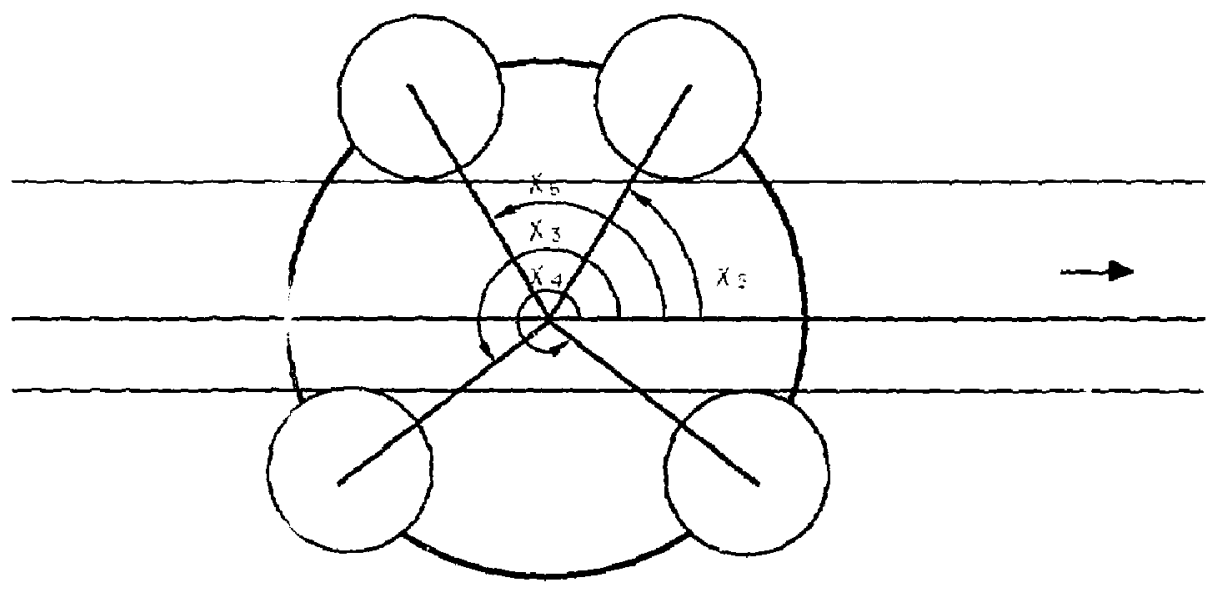

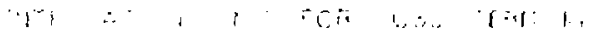




$$
0
$$


A. H. Futch - Eigure 4

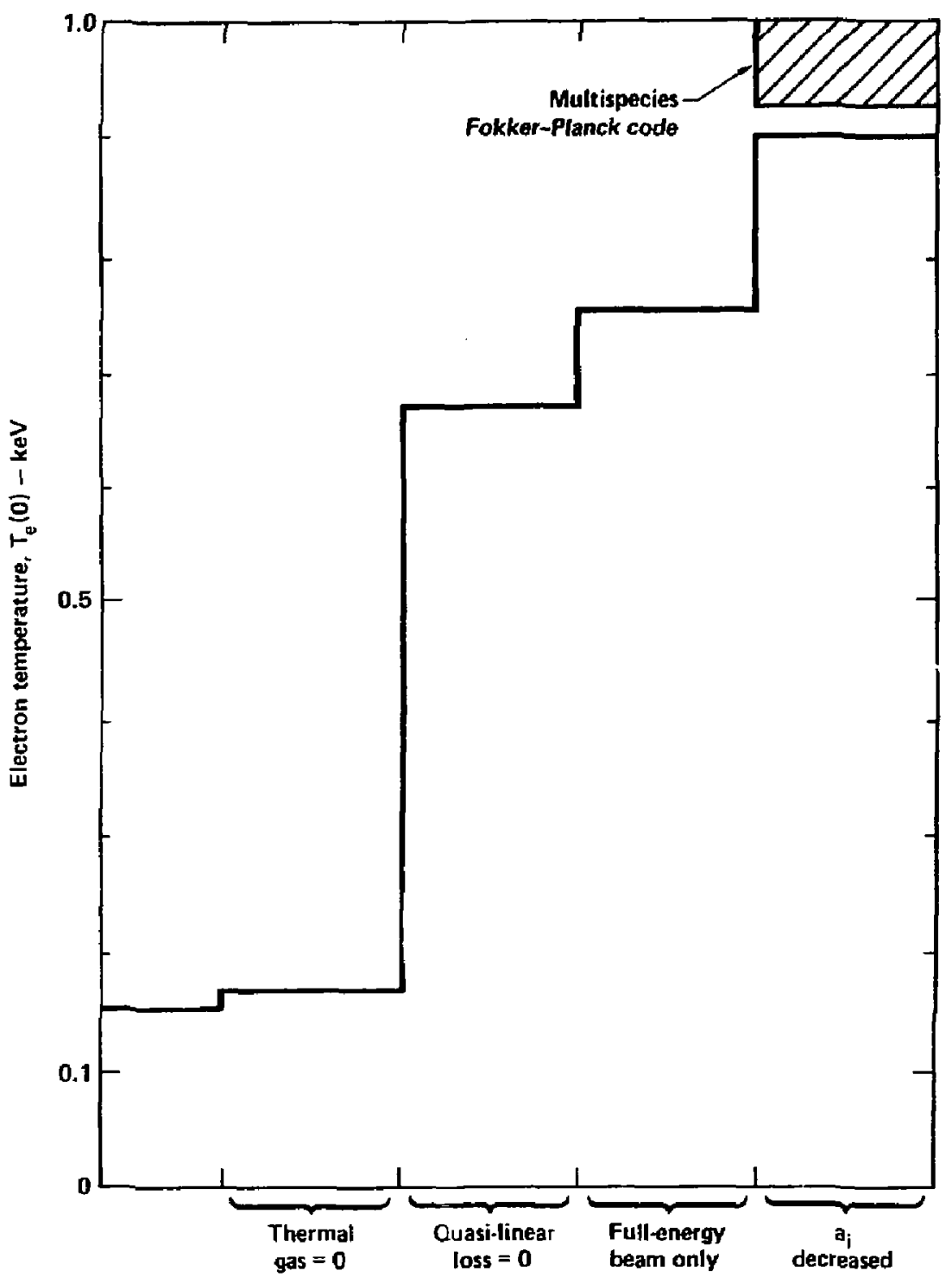


A. H. Futch - Figure 5

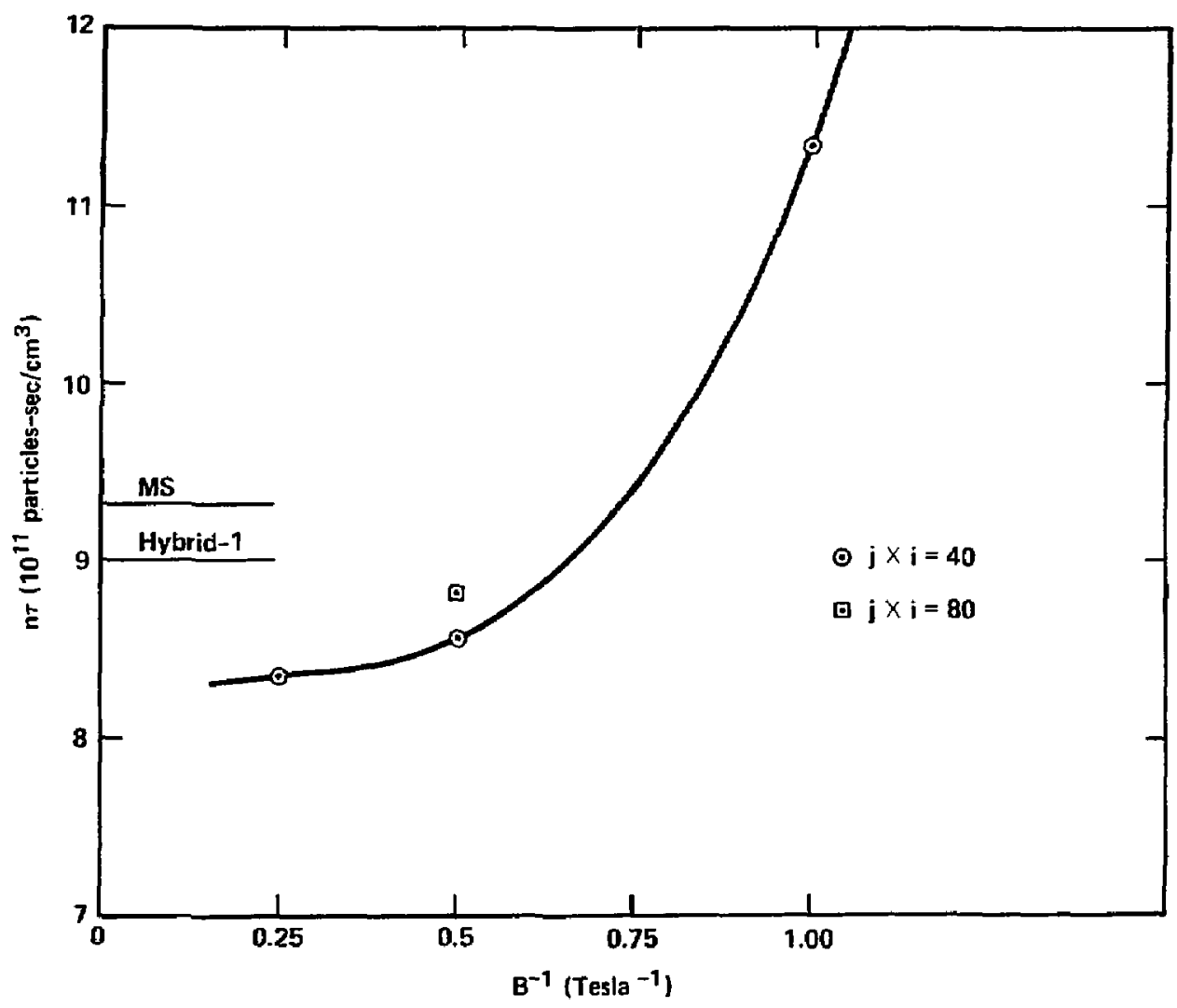


APPENDIX A

ARCHER FUTCH

Colorado Springs

APS

1978 
Quasilinear Diffusion and Electron Temperature Calculations in the 2XIIB Experiment--Archer H. Futch and Donald Freeman, Lawrence Livermore National laboratory--Electron temperature measurements in the 2XIIB experiment are much lower than predictions based on spatially constant Fokker-Planck calculations unless additional heat losses are included for electrons (1.e., cold electron stream). The present paper describes a spatially dependent Fokker-Planck model which glves $\mathrm{T}_{e}$ close to experimental values without Including streaming electrons. The calculated $T_{e}$ arops from the spatially constant Fokker-Planck value (1 keV) to approximately $150 \mathrm{eV}$ when quasilinear diffusion and finlte radius effects are included. The calculated $\mathrm{T}_{\mathrm{e}}$ is in good agreement with measurements when 2 XIIB is operated under optimum conditions. The computer results are easily understood in terms of a simple model which gives $T_{e} \propto \tau_{p}^{2 / 5}$ where $\tau_{p}$ is the particle lifetime. Radial diffusion was also observed to stabllize and prevent the decrease in plasma radius which wolld otherwise occur as a result of surface charge exchange with themal gas.

\section{DERIVATION OF FOKKER-PLANCK EQUATION}

Assumptions:

1) $B_{z}$ is uniform in $r$ and a square-well in $z$.

2) Collision frequency is much less than the gyrofrequency $v \ll \Omega$.

3) $v, R$, are constants of the motion 
$-A 2-$

$$
\frac{\partial f}{\partial t}+v_{r} \frac{\partial f}{\partial r}=\left(\frac{\partial f}{\partial t}\right)_{c}, \text { since } \dot{v}=\dot{R}=\dot{\theta}=0 .
$$

Expanding $f$ in terms of vaR: $f=f_{0}+f_{1}+\ldots$

and equating 1 like powers of $v / g$

lowest order $v_{r} \frac{\partial f_{0}}{\partial r}=0$

first order $\frac{\partial f_{0}}{\partial t}+v_{r} \frac{\partial f_{1}}{\partial r}=\left(\frac{\partial f_{0}}{\partial t}\right)_{c}$

Integrating around a gyro-orbit:

$$
\begin{aligned}
& \frac{\partial f_{D}}{\partial t} \oint \frac{d r}{v_{r}}+\oint \frac{\partial f_{1}}{\partial r} d r=\oint\left(\frac{\partial f_{0}}{\partial t}\right)_{c} \frac{d r}{v_{r}} \\
& \frac{\partial f_{0}}{\partial t}=\frac{1}{\tau} \oint\left(\frac{\partial f_{0}}{\partial t}\right)_{c} \frac{d r}{v_{r}}=\tau=\oint \frac{d r}{v_{r}}=\frac{2 \pi}{\Omega} \\
& =\frac{1}{\pi} \int_{R-\rho}^{R+\rho} \frac{\left(\frac{\partial f_{0}}{\partial t}\right)_{c} r d r}{\sqrt{4 R^{2}{ }^{2}-\left(r^{2}-R^{2}-\rho^{2}\right)^{2}}}
\end{aligned}
$$


Where we have used the cos law, 1.e., $r^{2}=R^{2}+\rho^{2}-2 R q \cos \beta$.

By separating varlables, the collision terms are modeled by an equation of the form

$$
\begin{aligned}
\frac{\partial D(R, v, t)}{\partial t}= & -\frac{1}{v^{2}} \frac{\partial}{\partial v}\left[v^{2}\left\langle\frac{\partial h}{\partial v}\right\rangle D(R, v, t)-\frac{v^{2}}{2}\left\langle\frac{\partial 2}{\partial v^{2}}\right\rangle \frac{\partial D(R, v, t)}{\partial v}\right]-\frac{\lambda}{2 v^{3}}\left\langle\frac{\partial B}{\partial v}\right\rangle D(R, v, t) \\
& +\left(A \frac{\partial}{\partial v}+\frac{B}{R} \frac{\partial}{\partial R}\right) D_{v}(R, v, t)\left(A \frac{\partial}{\partial v}+\frac{B}{R} \frac{\partial}{\partial R}\right) D(R, v, t) \\
& +K_{2}(R, v, t) \delta\left(v-v_{b}\right)-K_{1}(R, v, t) D(R, v, t)-D(R, v, t) \\
& \sigma_{c x} v\left\langle n_{0}(r)\right\rangle
\end{aligned}
$$

where $h(r, v)$ and $g(r, v)$ are the 1sotrople form of the "Rosenbluth potentials" and $\langle()>$ Indicates the phase average around the particle orbit

$$
\text { < })\rangle=\frac{2}{\pi} \int_{R-\rho}^{R+\rho} \frac{() r d r}{\sqrt{4 R^{2} \rho^{2}-\left(r^{2}-R^{2}-\rho^{2}\right)^{2}}}
$$

and

$$
D_{v}(R, v, t)=\sum_{m}\left(\frac{q \bar{\phi}}{M_{i}}\right)^{2} \frac{J_{m}^{2}\left(\frac{k_{i} v}{\omega_{c i}}\right)}{\Delta \omega}
$$


$K_{2}(R, v, t)$ are the collisional trapping terms and $K_{1}(R, v, t)$ corresponds to the loss of particles by resonance charge exchange with neutral beam atoms. The last term of Eq. $(A-1)$ results from the loss of ions by charge exchange with thermal gas molecules or atoms.

After solving Eq. $(A-1)$ for the guiding center density, $D(R, v, t)$, the real distribution function is obtalned by the transformation

$$
f(v, r, t)=\frac{2}{\pi} \int_{|r-\rho|}^{r+\rho} \frac{D(R, y, t) R d R}{\sqrt{4 R^{2} r^{2}-\left(R^{2}+r^{2}-\rho^{2}\right)^{2}}}
$$

the Ion density is calculated by

$$
n(r, t)=4 \pi \int f(v, r, t) v^{2} d v
$$

Pastukhov rate equations are solved for the electron density, $n_{e}(r)$, and temperat ure, $T_{e}(r)$

$$
\frac{d n_{e}}{d t}=-\frac{2}{\sqrt{\pi}} \frac{n_{e}^{2}}{n_{e} T} \frac{2 R_{m}}{2 R_{m}+1} \frac{e^{-\frac{e}{T} \phi}}{\ln \left(4 R_{m}+2\right)} \frac{T_{e}}{e \phi}\left[1+\frac{T e}{2 e \phi}-\frac{1}{4} \frac{T e}{e \phi}+\ldots\right]+J_{e}
$$




$$
\frac{d\left(n_{e} E_{e}\right)}{d t}=\text { (energy transfer from lons) }-\frac{\left(\phi+T_{e}\right) n^{2}}{n_{t}}+J_{e} \cdot E_{e}^{0}
$$

The plasma potential, $\phi(r, t)$ is computed at each time step by requiring charge neutrality. 
-AG-

ENERGY AND MAGNETIC FIELD SCALING OF QUASILINEAR AND CHARCE-EXCHANCE DIFFUSION TERMS

$$
\frac{\partial F}{\partial t}=\frac{A^{2}}{v^{2}} D_{v} \frac{\partial^{2} F}{\partial v^{2}}+\frac{B^{2}}{r^{2}} D_{v} \frac{\partial^{2} F}{\partial r^{2}}+D e x \frac{\partial^{2} F}{\partial r^{2}}
$$

$\propto \frac{\omega_{c I}}{v^{2}}-D_{v} \frac{\partial^{2} F}{\partial v^{2}}+\frac{k^{2}}{\omega_{c 1}^{2}} D_{v} \frac{\partial^{2} F}{\partial r^{2}}+\frac{\sigma_{c x}^{A} E_{1}}{B^{2}} \frac{\partial^{2} F}{\partial r^{2}}$

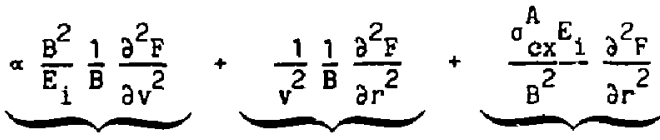

End-loss Q.L. Radial ex Radial

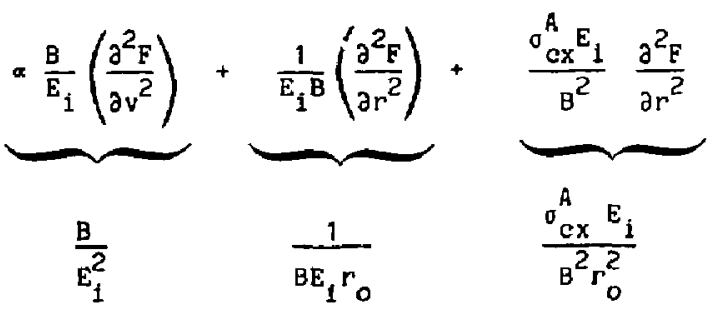

where $D_{c x}=J_{c x}^{A} \rho^{2 / 4}$

and $\rho^{2}+E_{i} / B^{2}$ 
CALCULATED GLOBAL POWER BALANCE FOR HEAD-ON INJECTION WITH 450 AMPS IN THE 2XIIB EXPERIMENT

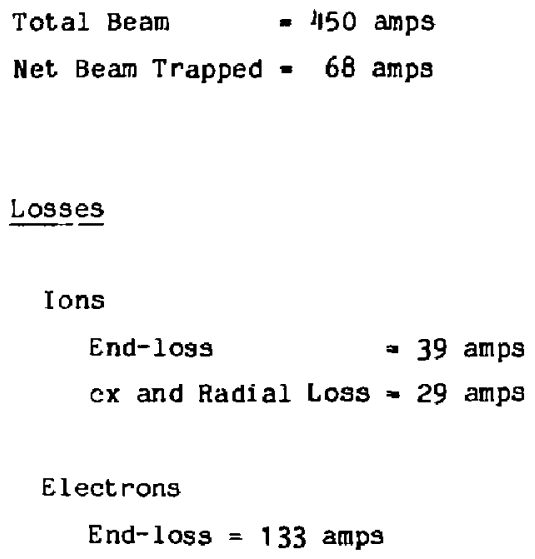

Power Trapped

$$
\text { Power }=\frac{68 \times 6.25 \times 10^{18} \times 15 \times 1.602 \times 10^{-9}}{10^{7}}
$$

$=1 \mathrm{MH}$

End-loss $=82$ kilowatts

$c x$ and Radial Loss $=918$ k1lowatts

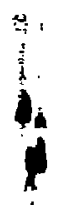

The ion beam current and lon losses correspond to energetic ions only. Since the electron losses also include the ionization crermal gas, the electron losses are greater than the ion losses. 
RESULTS

Comparisons have been made between measured 2 XIIB plasma parameters and calculated parameters for a large radius plasma $(12 \mathrm{~cm})$ with head-on injection. The addition of quasilinear (Q.L.) diffusion to the basic Fokker-Planck code causes major changes in plasma parameters since Q.L. loss rates are much greater than coulomb loss rates ( -50 times greater) at equilibrium densities. A brief summary of some of the observations are the following:

1. Radial profiles were changed significantly when Q.L. diffusion was present. In previous runs without Q.L. diffusion the plasma decreased In radius as thermal gas eroded away the plasma surface. With Q.L. diffusion present the surface was stabilized by diffusion that moved the excess fons trapped in the plasma interior to the surface to compensate for the large charge exchange losses of the surface. (See pages 4 and 5. )

2. Calculated values of plasma density and electron temperature are in agreement with measurements under optimum conditions. (See page 6.)

3. The effect on the electron temperature at the plasma center of removal of various physical effects from the code. (See page 7.)

4. The following very slmple model for the electron temperature was developed to describe results fron the code: 


$$
\frac{d\left(n_{e} E_{e}\right)}{d t}=n\left(\frac{w_{i}}{\tau_{1 e}}\right)-\left(\phi+T_{e}\right) \frac{n^{2}}{n \tau} .
$$

At equilibrium

$$
\begin{aligned}
& \frac{n_{e}}{n}=\frac{\left(\phi+T_{e}\right)}{\left(\frac{w_{1}}{\tau_{i e}}\right)}=\frac{C T_{e}^{5 / 2}}{n W_{1}} \quad c=\text { constant } \\
& T_{e}=c^{-2 / 5}\left(n W_{1} \tau_{i}\right)^{2 / 5}
\end{aligned}
$$

In the plasma interior $\tau_{e}$ is equal to the hot ion lifetime, $\tau_{i}$, and

$$
T_{e} \propto\left(n w_{1} T_{1}\right)^{2 / 5}
$$

The strong dependence of the Ion lifetime with the magnetic field determines the broad features of 2 XIIB plasma magnetic field scaling. Radial losses were found to dominate below 0.7 Tesla. The loss rates versus magnetic field, $B$, are shown on page 8 .

5. Page 9 shows a comparison of the calcuiated $T_{e}$ scaling, as compared with the experimental data for $10 / 25 / 7 i$ and $10 / 20 / 77$. Only shots which did not include guns $\# 3$ and $\# 5$ which pass chrough the plasma center are plotted. 
6. Page 10 shows the line integral ion energy vs. B for both code results and measured data. The lon energy at the plasma center is also shown.

7. Since radial losses scale as either $p$ (Q.L.) or $p^{2}$ (ex difrusion), the higher velocity particles have a shorter lifetime. This energy dependence explains the decreasing average ion energy as $\theta$ decreases shown on page 10. Page 11 shows the effect of decreasir., $B$ on the veloeity distribution function. 


\section{APPENDIX A FIGURE CAPTIONS}

Flgure A1. See Flgure 1 of maln text.

F1gure A2. Calculated electron energy and denslty versus radius for head-on injection in the 2XIIB experiment and with quasilinear diffusion terms. Non-equiliorium results at $1.4 \mathrm{msec}$. Plasma radius stabilized by diffusion that moved the excess ions trapped in the plasma interior to the surface to compensate for large charge-exchange losses. Diffusion stabilizes the surface boundary.

Figure A3. Calculated ion energy and density versus radius for head-on injection in the $2 X I I B$ experiment and with quasilinear diffusion terms. Non-equilibrium results at $1.4 \mathrm{~m} \mathrm{sec.} \mathrm{Diffusion}$ stablilzas the surface boundary.

Pigure A4. Calculated electron energy and density versus radius for head-on injection in the 2XIIB experiment and without quasilinear diffusion terms. Non-equilibrium results at 1.3 msec. Decreasing plasma radius results from thermal gas erosion at the plasma surface.

Figur'e A5. Calculated ion energy and density versus radius for head-on injection in the $2 X I I B$ experiment and without quasilinear diffusion terms. Non-equilibrium results at 1.3 msec. Decreasing plasma radius results from thermal gas erosion at the plasma surface. 
Figure A6. Comparison of code results with experimental data for the 2XIIB experiment.

F1gure A7. See Figure 4 of maln text.

Figure A8. Calculated loss rates for the 2XIIB experiment at the plasma center versus magnetic fleld.

Figure A9. Electron temperature scaling versus magnetle field for the $2 X I I B$ experiment.

Figure A10. Average ion energy versus magnetic field for the $2 X I I B$ experiment.

Figure A11. Calculated distribution function versus magnetic field for the ¿XIIB experiment.

Figure A12. Measured distribution function for 2XIIB versus magnetic field. Higher energy components decrease as the magnetic field decreases. 
$-A 13-$

A. H. Futch - Figure A1

See Elgure 1 of main text. 
A. H. Futch - Figure A2

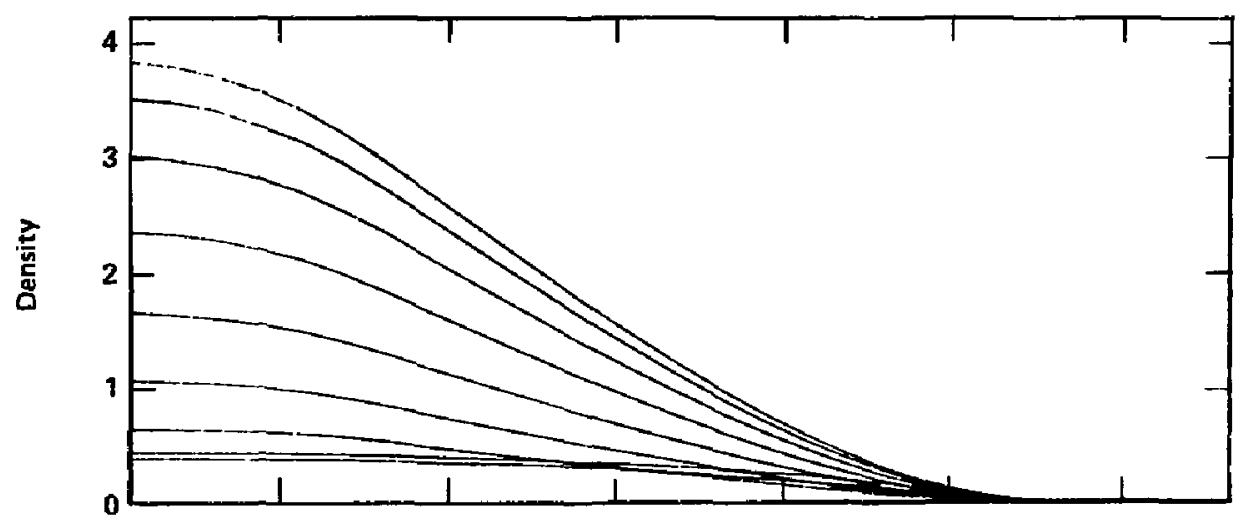

$E+13$

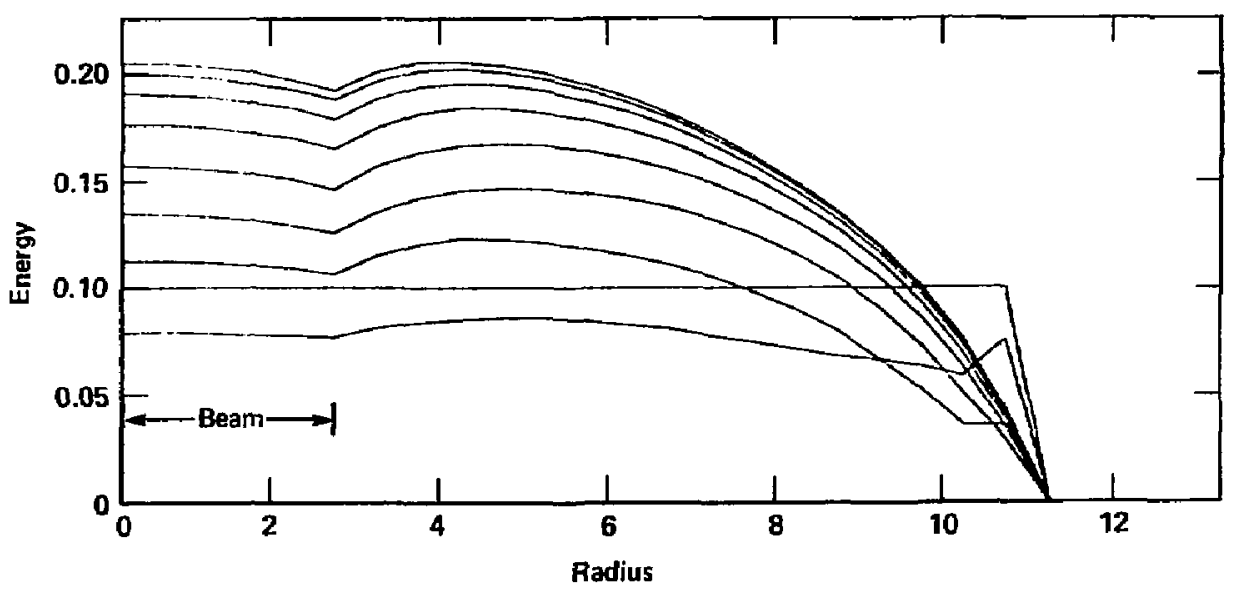


A. H. Fut cit [igurid
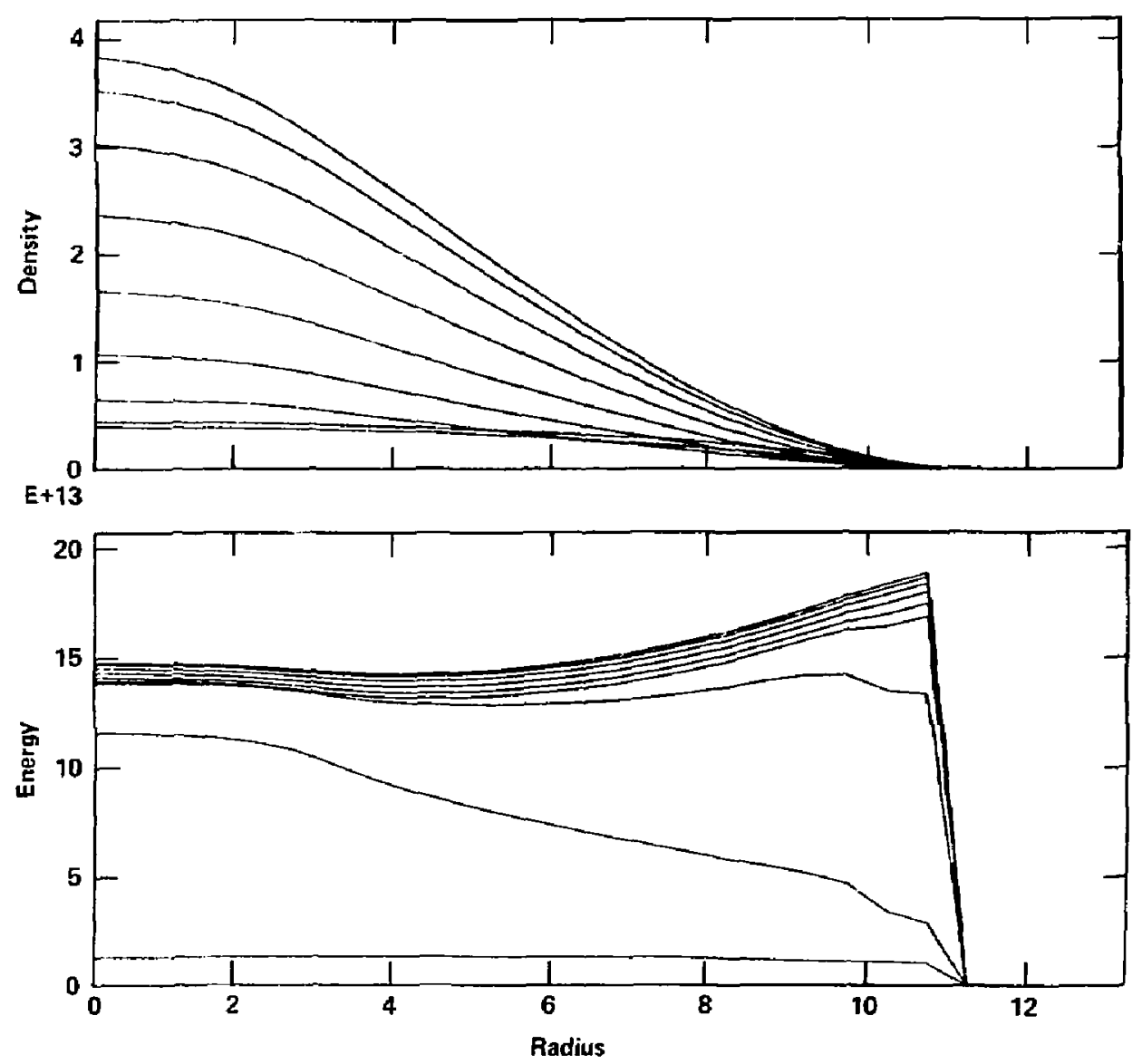
A. H. Futch - Eigure A4
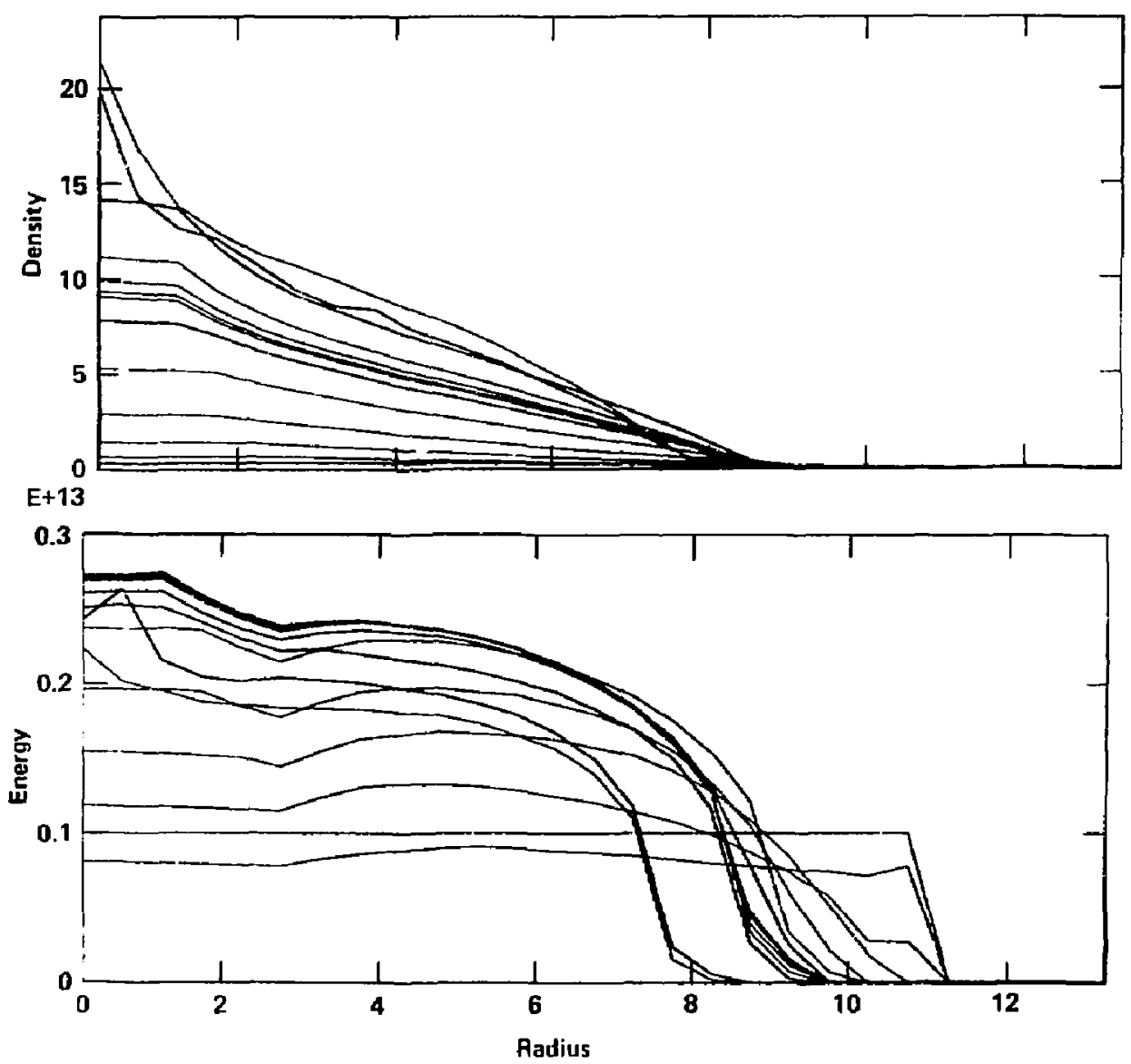
A. 4 Futa' - I:igur $f$;

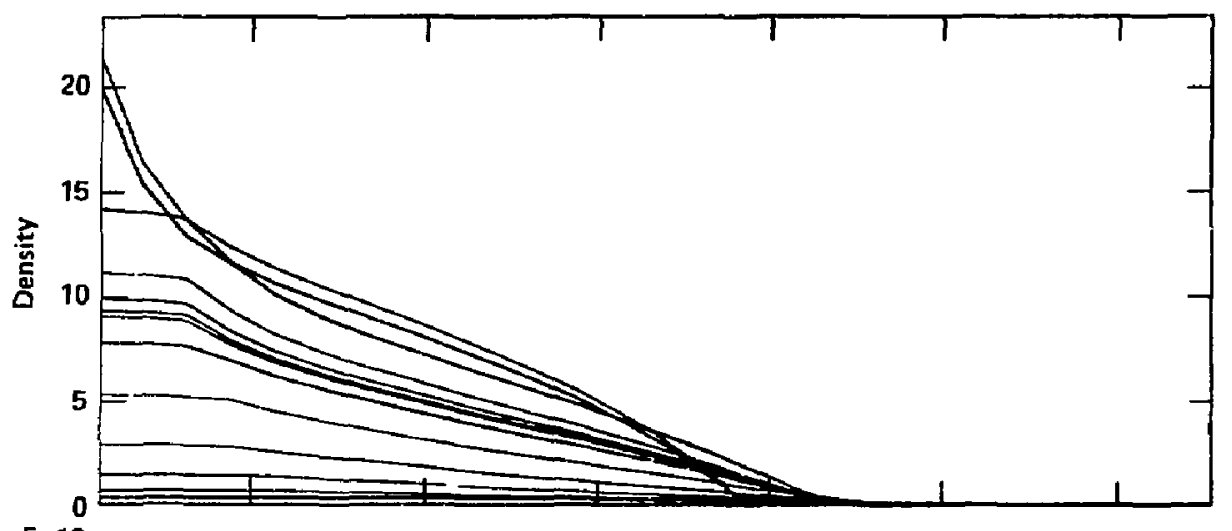

$E+13$

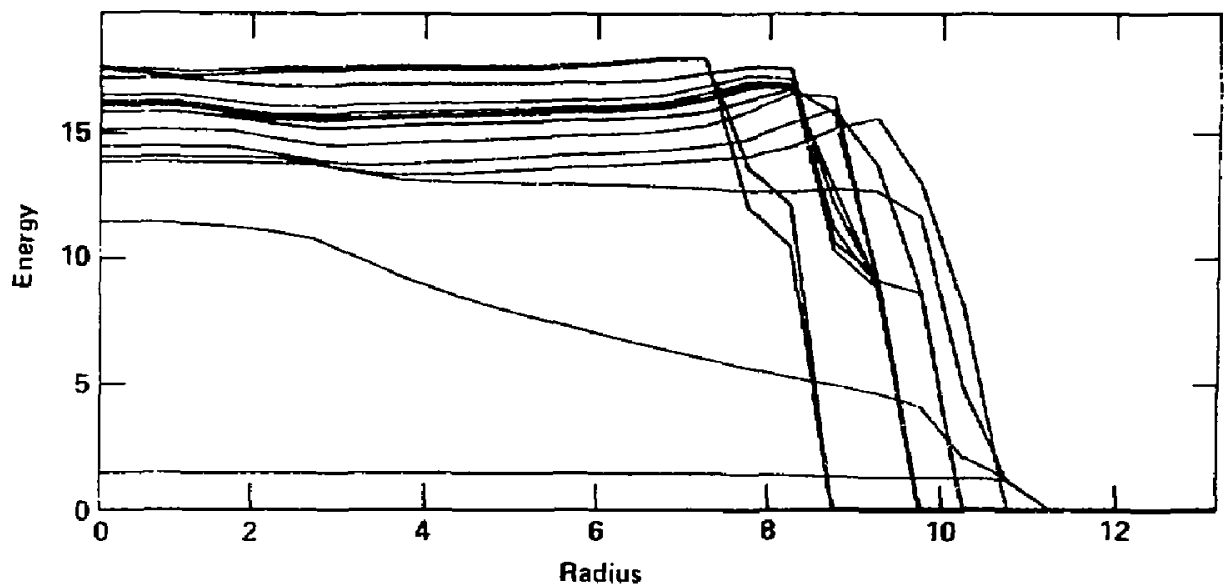


A. H. Futch - Eigure $A 6$

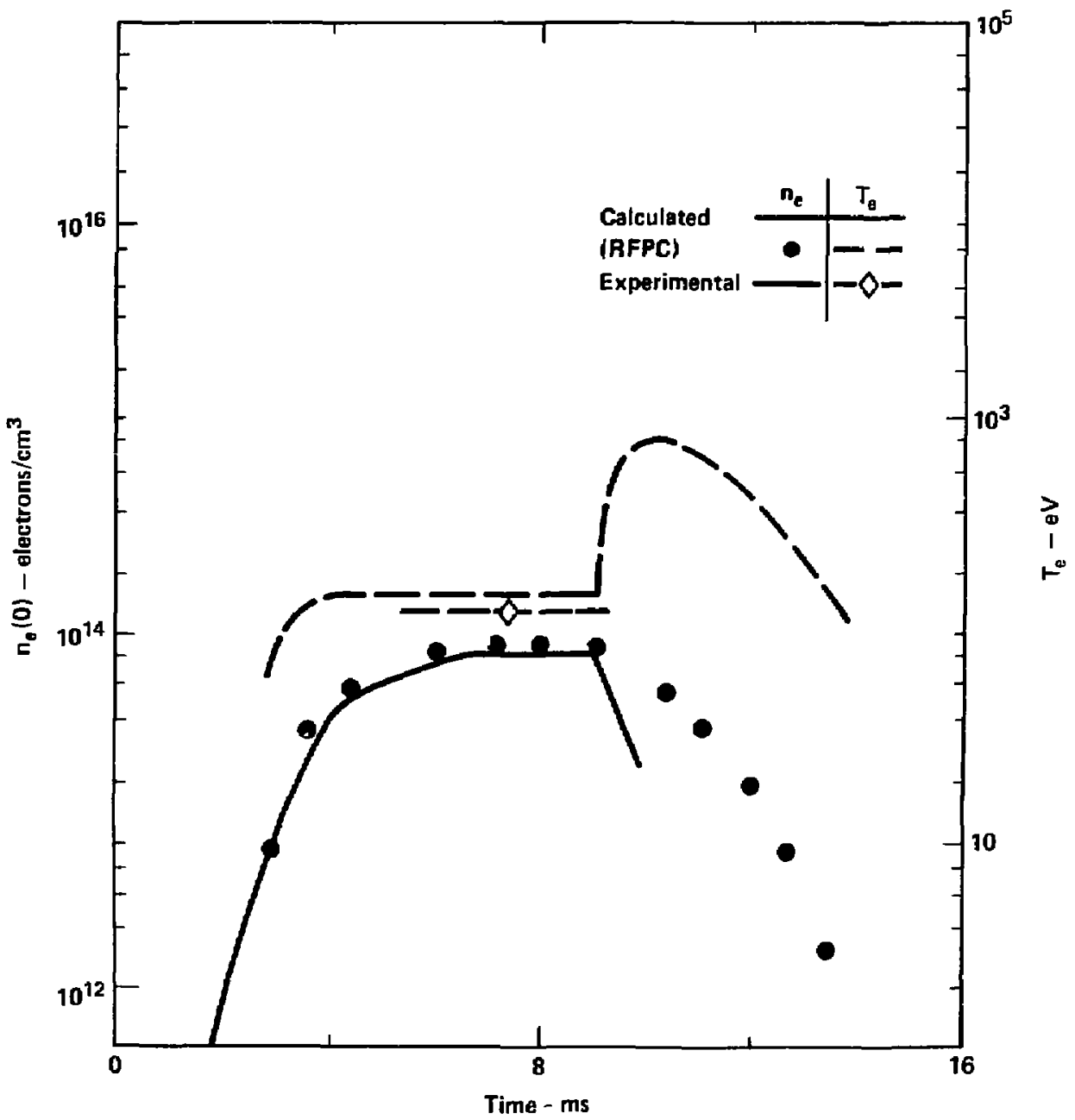


-A19-

A. :H. Futch - F1gure A7

See Figure 4 of maln text. 
A. Hi. Fulsh - ligure ais

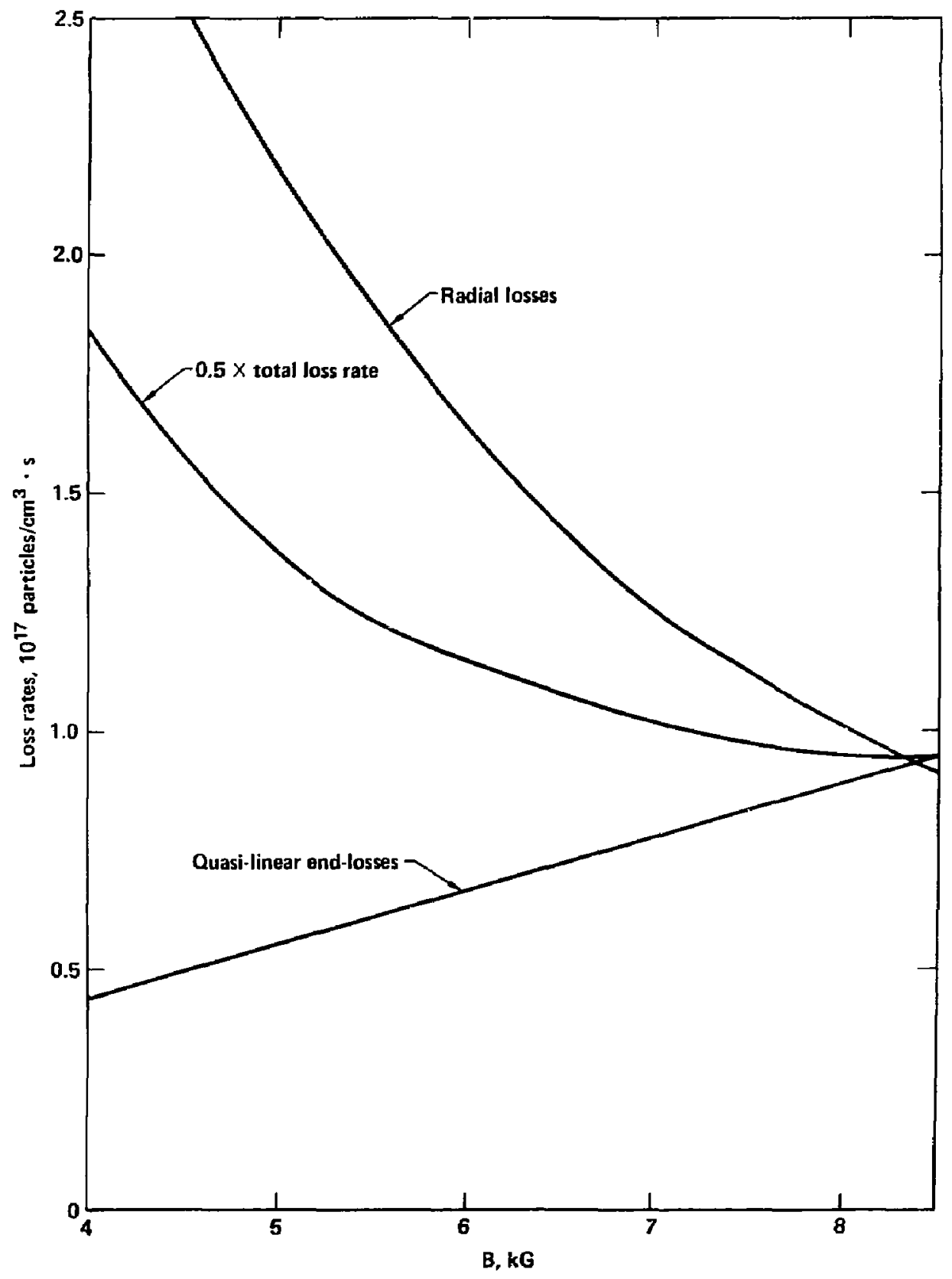


A. H. Fulch - Figure Ai

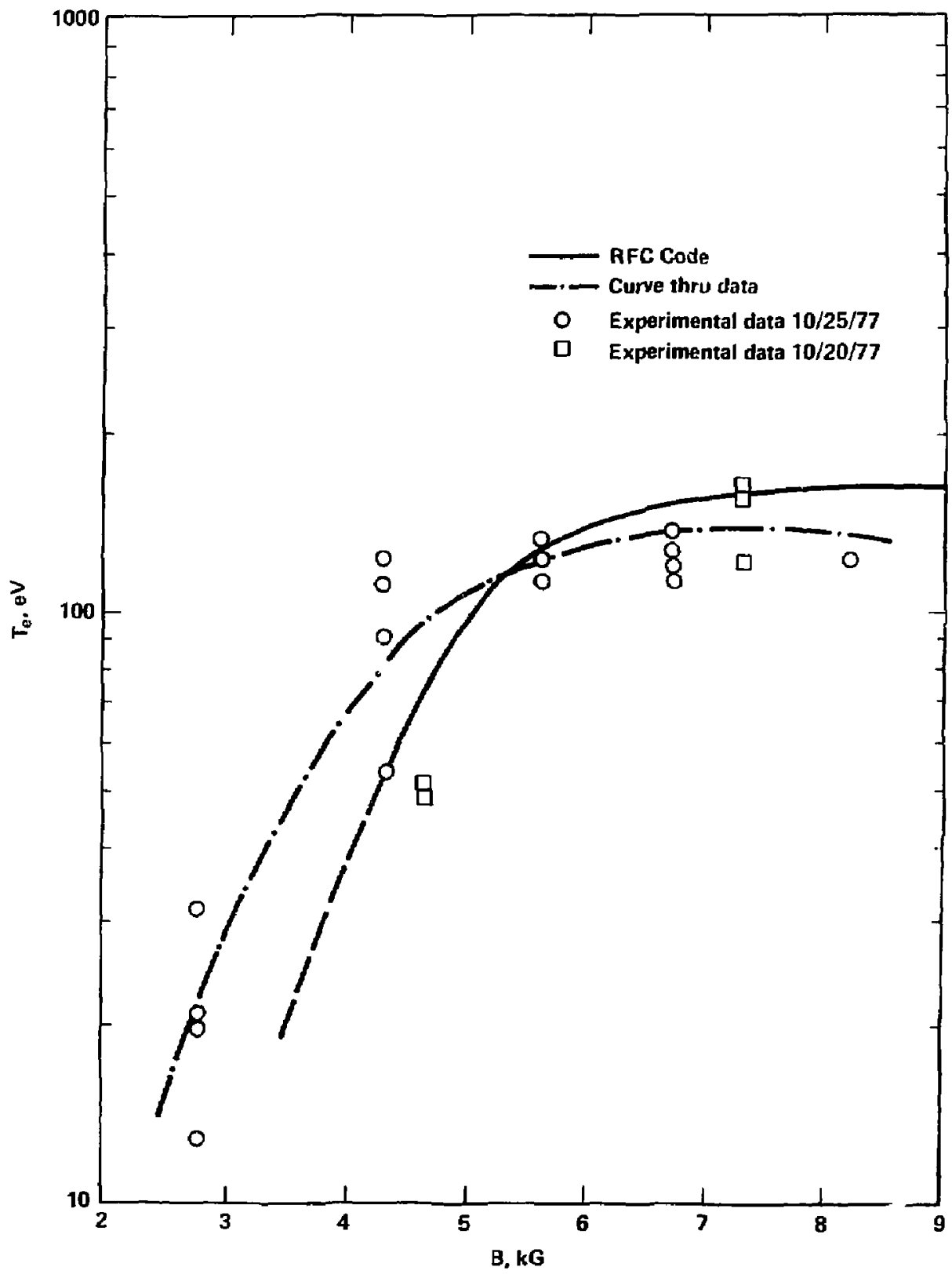


A, H. Futch - Figure A10

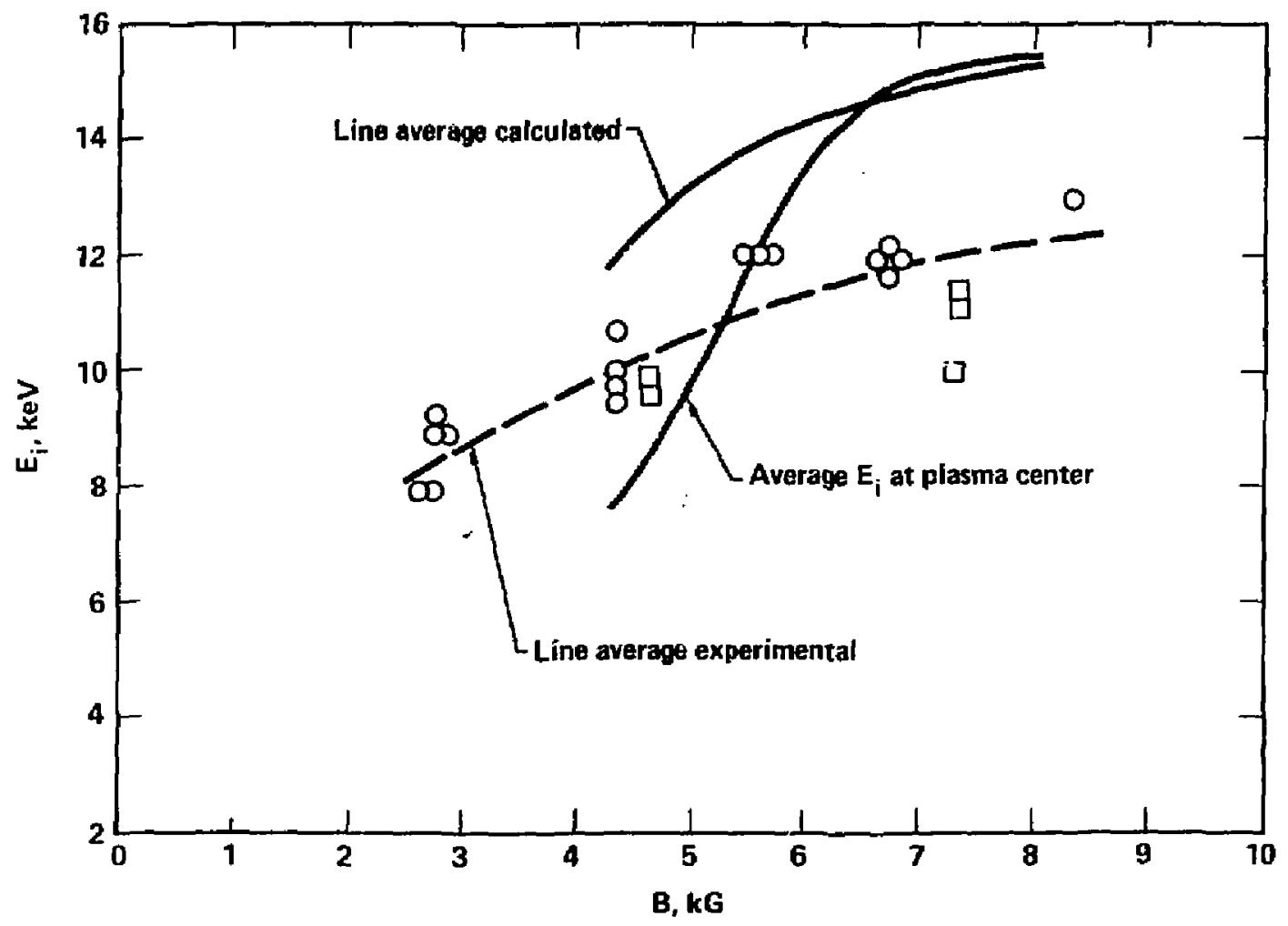


A. H. Futch - F1gure A11
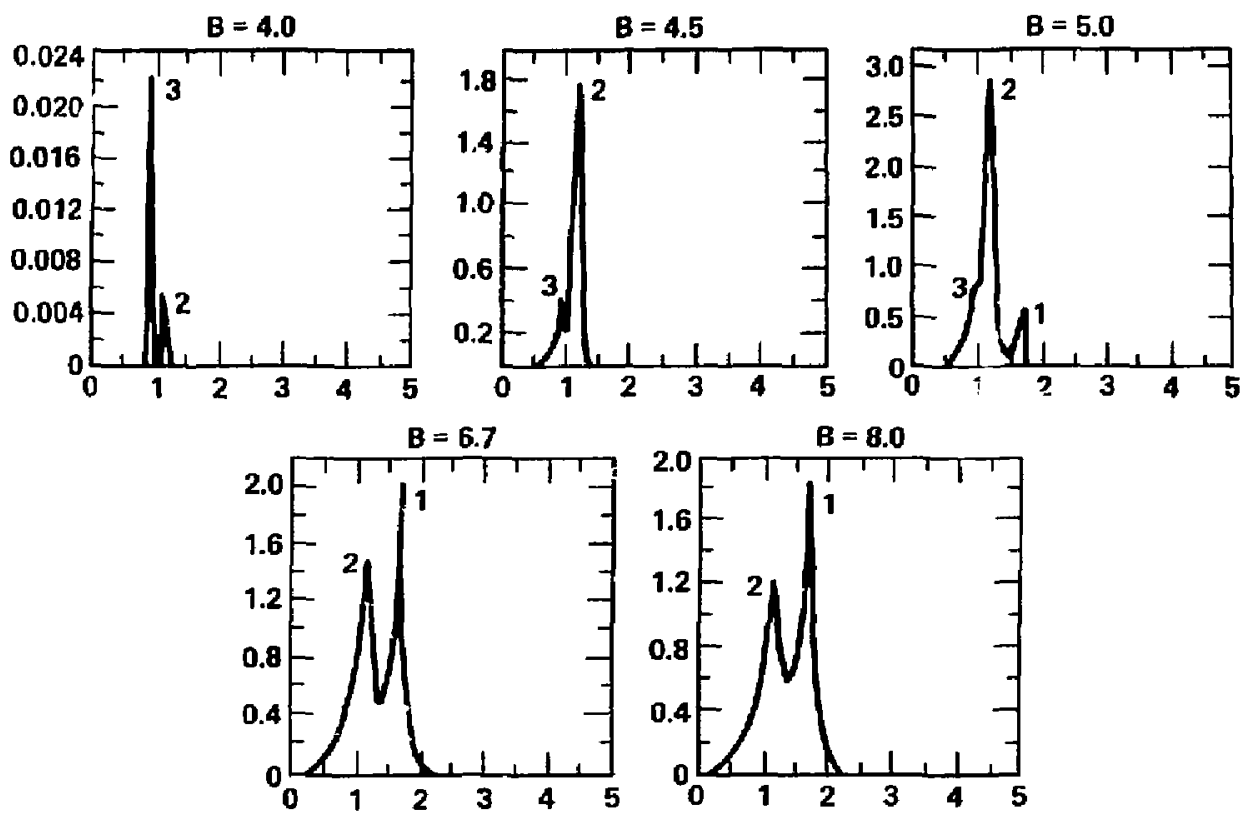
A. H. Futch - Figure $\mathrm{A} 12$
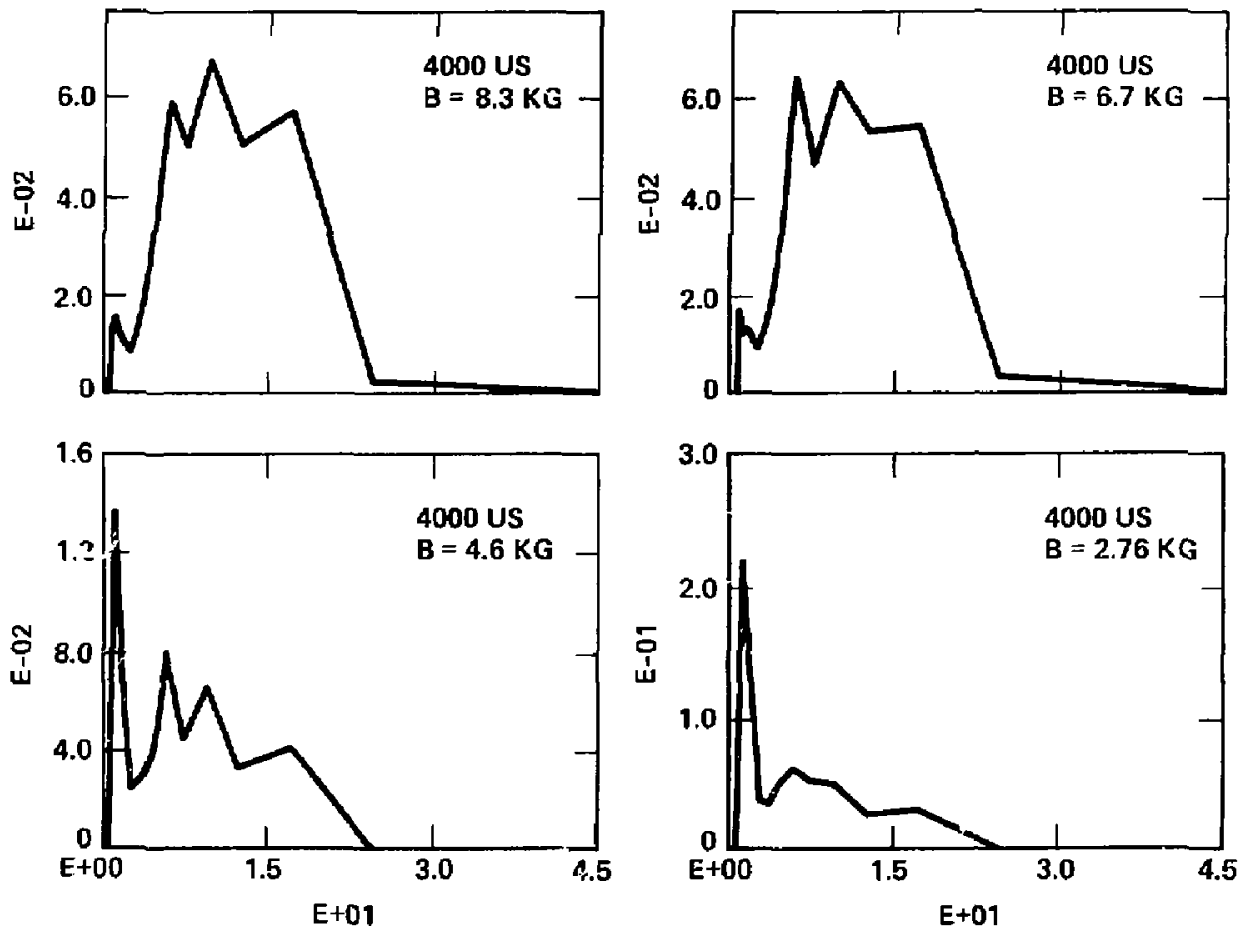


\title{
APPENDIX B
}

MEASURED PLASMA LIFETIME VERSUS

RADIUS FROM 2XIIB DATA

\begin{abstract}
The radial dependence of the plasma lifetime is calculated from measurements of the radial and axlal density profile, the plasma density, and the fraction of the neutral beam trapped. The plasma density is given by

$$
n(r, z)=\hat{n} \exp \left[-\left(n / R_{p}\right)^{2}-\left(z / L_{p}\right)^{2}\right]
$$
\end{abstract}

The plasma lifetime is calculated under equilibrium condition by

$$
J(r)=\frac{n(r)}{\tau(r)} \quad T(r)=\frac{n(r)}{J(r)}
$$

or

$$
n \tau(r)=\frac{n(r)^{2}}{J(r)}
$$

where $J(r)$ is the current trapped per co by jonizing collisions. The geometry dependent factor of the trapped current is obtained from a computer 
code using the measured density profile and measured fraction of the neutral beam trapped. Figure $B 1$ shows the results of the calculation for head-on Injection (Case 2 of "Energy Confinerent in Mirror Plasmas Versus Radial Size"). The calculation assumes a rectangular shape for the beam instead of gaussian. If a gausstan shape were assumed, then the flat part of the curve near the center would tend to disappear.

If electron drag dominated hot ion losses, then nt would be constant as a function of radius if $T_{e}$ were constant. The increase of $n \tau$ with radius is inconsistent with the measured radial dependence of $\mathrm{T}_{\mathrm{e}^{*}}$ we therefore conciude that electron drag is not the dominate hot ion loss process (at least for this case).

At first glance, it seems surprising that the strong dependence shown in Fig. Bi should occur since a straightforward application of the electron drag formula gives $T$ (drag) $\approx \tau$ (measured); however, a straightforward application of the drag formula is probably not valfd since a plasma ion makes many charge exchange collisions with the beam during a drag time Nexsen and Rensink effect. (This is especially true near the plasma center.) Each charge exchange collision returns the particle to the injected beam energy and angle, thereby reducing the effect of electron drag. On the other hand a particle diffusing radially by quasilinear scattering moves further out by charge exchange with the beam.

Paul Drake has pointed out (MFE/CP/78-483) that the electron lifetime necessary to give typical electron temperature is $=25 \mu \mathrm{sec}$. Therefore, the contribution of radial losses to $\mathrm{T}_{\mathrm{e}}$ scaling can be neglected for typical 
2XIIB plasmas. Optimum electron temperatures may be a factor of 2 higher than typical electron temperatures. The electron lifetime is then $=(2)^{5 / 2}$ - 6 times longer. If the magnetic fleld is thus varied to obtain $T$ versus $R$ data ( $C$ lauser's dsta), then the Ion Iffetime appears necessary to explain the observed $T_{e}$ dependence shown in Figure B2. The particle lifetime scalling should include radial 10 s terms. 
$-\mathrm{B} 4-$

APPENDIX B FICURE CAPTIONS

Flgure Bl. nt and $\tau$ versus radius for head-on injection--Case 2 of energy confinement paper.

F1gure B2. Electron temperature scaling versus magnetlc fleld. 
A. H. Futch - Figure B1

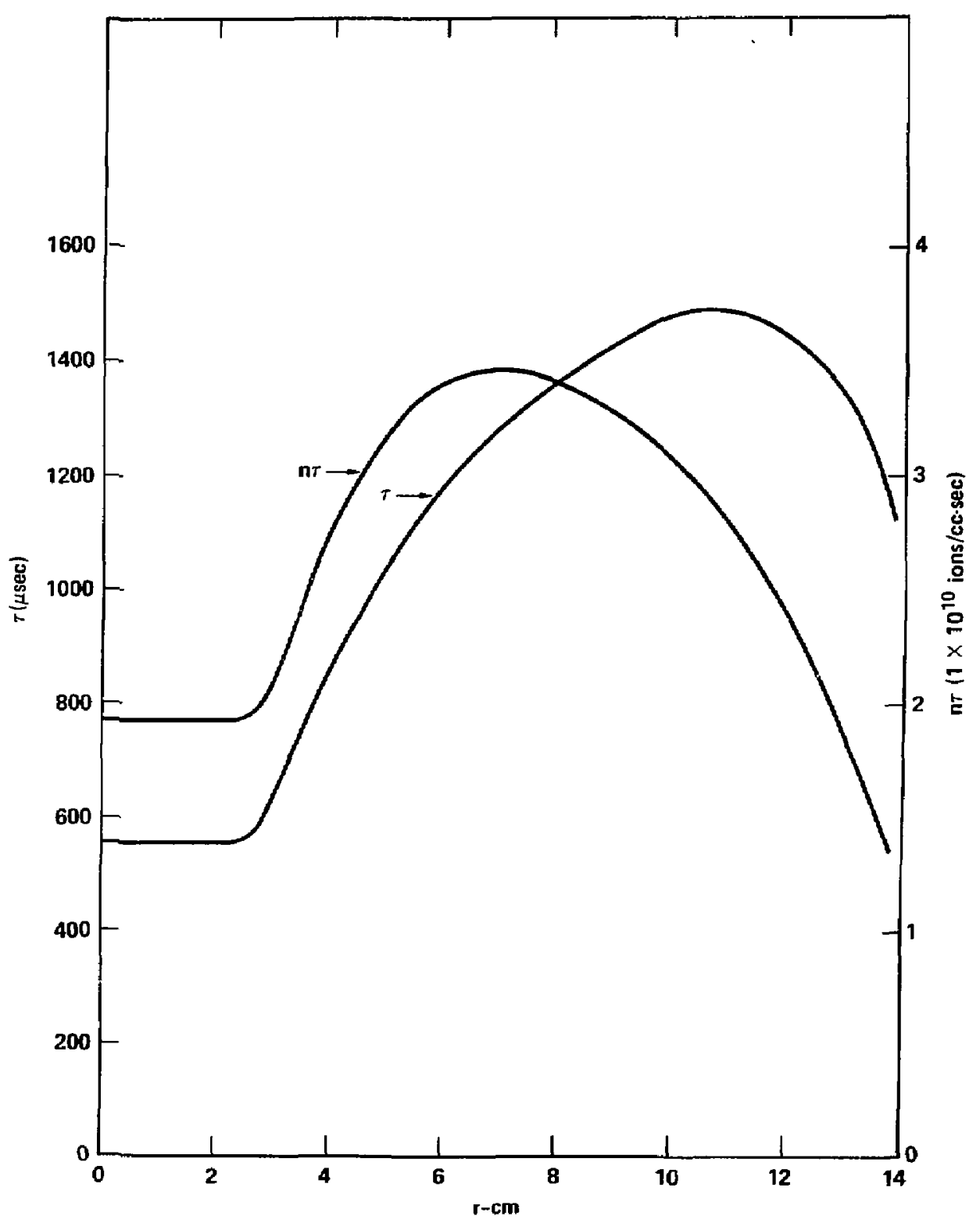


A. H. Futch - Figure 82

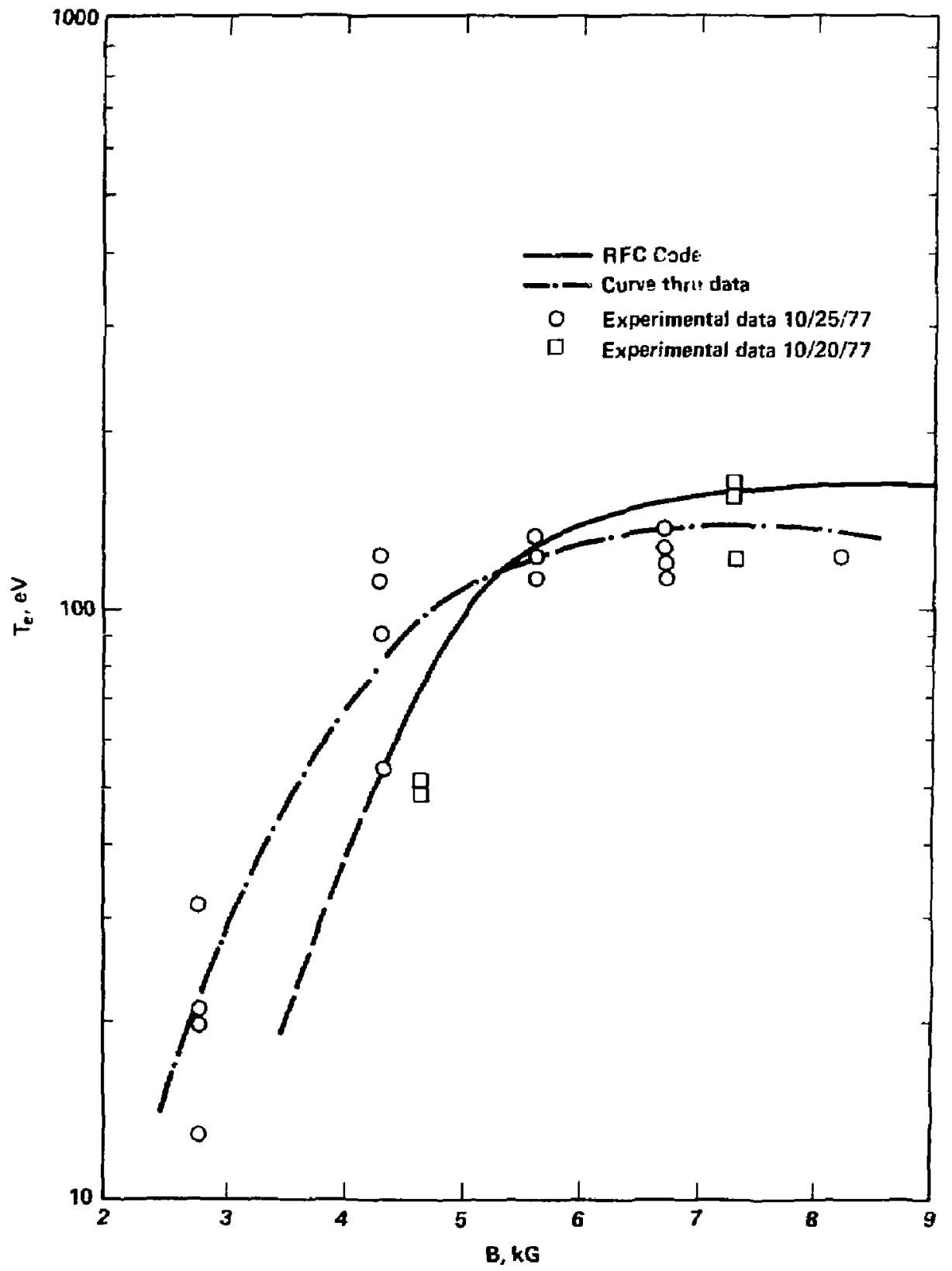

